\title{
One-dimensional bargaining with Markov recognition probabilities
}

Citation for published version (APA):

Herings, P. J. J., \& Predtetchinski, A. (2010). One-dimensional bargaining with Markov recognition probabilities. Journal of Economic Theory, 145(1), 189-215. https://doi.org/10.1016/j.jet.2009.10.002

Document status and date:

Published: 01/01/2010

DOI:

10.1016/j.jet.2009.10.002

Document Version:

Publisher's PDF, also known as Version of record

Document license:

Taverne

Please check the document version of this publication:

- A submitted manuscript is the version of the article upon submission and before peer-review. There can be important differences between the submitted version and the official published version of record.

People interested in the research are advised to contact the author for the final version of the publication, or visit the DOI to the publisher's website.

- The final author version and the galley proof are versions of the publication after peer review.

- The final published version features the final layout of the paper including the volume, issue and page numbers.

Link to publication

\footnotetext{
General rights rights.

- You may freely distribute the URL identifying the publication in the public portal. please follow below link for the End User Agreement:

www.umlib.nl/taverne-license

Take down policy

If you believe that this document breaches copyright please contact us at:

repository@maastrichtuniversity.nl

providing details and we will investigate your claim.
}

Copyright and moral rights for the publications made accessible in the public portal are retained by the authors and/or other copyright owners and it is a condition of accessing publications that users recognise and abide by the legal requirements associated with these

- Users may download and print one copy of any publication from the public portal for the purpose of private study or research.

- You may not further distribute the material or use it for any profit-making activity or commercial gain

If the publication is distributed under the terms of Article $25 \mathrm{fa}$ of the Dutch Copyright Act, indicated by the "Taverne" license above, 


\title{
One-dimensional bargaining with Markov recognition probabilities
}

\author{
P. Jean-Jacques Herings ${ }^{*, 1}$, Arkadi Predtetchinski ${ }^{1}$ \\ Department of Economics, Maastricht University, PO Box 616, 6200 MD Maastricht, The Netherlands
}

Received 23 October 2008; accepted 8 September 2009

Available online 8 October 2009

\begin{abstract}
We study a process of bargaining over alternatives represented by points in the unit interval. The paper focuses on the asymptotic behavior of the subgame perfect equilibrium in stationary strategies as the continuation probability approaches one. We give a complete characterization of the limit of the equilibrium proposals as the generalized fixed point of the decumulative distribution of the players' ideal points as induced by the recognition probabilities. In contrast to the existing literature, we find no general relationship between the limit equilibrium proposals and either the Nash bargaining solution or the median voter outcome.
\end{abstract}

() 2009 Elsevier Inc. All rights reserved.

JEL classification: $\mathrm{C} 78$

Keywords: One-dimensional bargaining; Markov process; Median voter theorem; Nash bargaining solution

\section{Introduction}

We consider the situation where a group of players has to choose an alternative out of a set of alternatives represented by points in a one-dimensional space. This problem features prominently in the literature on collective decision making and typical examples concern the choice of the taxation level, the location of a facility, or the amount of money devoted to a particular investment

\footnotetext{
* Corresponding author. Fax: +31433884878 .

E-mail addresses: P.Herings@maastrichtuniversity.nl (P.J.J. Herings), A.Predtetchinski@maastrichtuniversity.nl (A. Predtetchinski).

1 The authors would like to thank the Netherlands Organization for Scientific Research (NWO) for financial support.
} 
opportunity. A famous solution within this setting is the median voter result (Black [4]). This result establishes conditions under which the chosen alternative corresponds to the one most preferred by the player with median preferences.

As in Banks and Duggan [1], Imai and Salonen [15], Cho and Duggan [8], Kalandrakis [16, 18], and Cardona and Ponsatí [6], we analyze the situation from a bargaining perspective. Problems in collective decision making are often solved by bargaining. Politicians bargain about public good levels, tax rates, and issues in the traditional left-right spectrum in political decision making. Within firms bargaining takes place by executives to choose among a variety of investment opportunities. Unions have to aggregate heterogeneous member preferences, which may involve bargaining, and next bargain with firms on terms of employment. These bargaining problems often involve a one-dimensional set of alternatives.

Banks and Duggan [1] consider bargaining over a set of social outcomes equal to an arbitrary compact convex subset of a Euclidean space. They examine a bargaining protocol where the proposer is selected according to a time-invariant probability distribution and they consider a general voting rule that determines whether a particular proposal is accepted or not. They prove the existence of a subgame perfect equilibrium in stationary strategies.

We restrict attention to the case where the set of alternatives is the unit interval and where acceptance of a proposal requires unanimity of all the players involved in the decision making process. The process by which the proposer is selected follows a general Markov process as in Kalandrakis [16]. The probability by which a certain player is selected as proposer is also referred to as the recognition probability.

We would like to emphasize the importance of a general model for the proposer selection process. Romer and Rosenthal [27] is among the most influential papers emphasizing the role of proposal making on the selected alternative. Kalandrakis [17] shows in a bargaining framework that the proposer selection process is more important than voting rights, impatience, or complex equilibrium strategies in explaining political power. Empirical support for this feature in the context of the allocation of transportation funds in the US is provided by Knight [19].

Two important special cases of our proposer selection model are the ones where the proposer is selected according to some time-invariant probability distribution as in Banks and Duggan [1] and Kalandrakis [18], or where the proposer is chosen according to some deterministically rotating scheme as in Cardona and Ponsatí [6]. Also the alternating offer bargaining model in Rubinstein [28] is an example of a deterministically rotating scheme and is a special case of our more general model. Merlo and Wilson [22] have generalized the Rubinstein set-up substantially and allow for a proposer selected by a Markov process. But since they consider bargaining problems where the dimension of the bargaining space equals the number of players minus one, one-dimensional bargaining with more than two players is not covered by their analysis.

The bargaining process we study proceeds as follows. At the beginning of a period one player is selected by means of a Markov process to make a proposal. The players react to the proposal sequentially and can each either accept or reject the proposal. If all players accept, the proposal is implemented, the game ends, and each player receives the payoff of the chosen alternative. Otherwise, with some probability negotiations break down and some disagreement outcome results, or, with the remaining probability, the next period begins.

We study subgame perfect equilibria in stationary strategies. In a stationary strategy, each player makes a unique history-independent proposal and has a unique proposer-dependent acceptance set. The acceptance set specifies which proposals are accepted by a player. The intersection of all the individual acceptance sets is called the social acceptance set. Like the individual acceptance set, the social acceptance set is proposer-dependent, but otherwise history-independent. 
We show that subgame perfect equilibria in stationary strategies are essentially unique. All equilibria have the same equilibrium proposals, the same equilibrium payoffs, and the same social acceptance sets. In equilibrium, proposals are immediately accepted, so in equilibrium no delay occurs. Any two equilibria can only differ with respect to the individual acceptance sets. The difference is only relevant off the equilibrium path and may occur when a player is indifferent between accepting or rejecting a particular proposal. The unique subgame perfect equilibrium in stationary strategies where players accept if they are indifferent between rejecting and accepting is referred to as the bargaining equilibrium.

Our uniqueness result complements the uniqueness results by Cho and Duggan [8] and Cardona and Ponsatí [6] in related frameworks, and the uniqueness result in Merlo and Wilson [23] for the extension of Rubinstein [28] to the $n$-person case that involves a stochastic cake under a contraction condition. Eraslan and Merlo [11] show for the latter model that uniqueness does not generally hold when approval of a proposal is not required to be unanimous. Eraslan [10] obtains a uniqueness result in the legislative bargaining approach of Baron and Ferejohn [2]. Kalandrakis [18] establishes a number of determinacy results (the number of equilibria is finite, and under some assumptions odd) for a quite general bargaining model.

We continue by studying the asymptotic behavior of subgame perfect equilibrium proposals as the continuation probability converges to one. As an illustration, consider the special case of time-invariant recognition probabilities. These recognition probabilities give rise to a cumulative distribution function on the set of alternatives, by assigning to each alternative the mass of players whose most preferred alternative is less than or equal to that alternative. We prove that in the limit the bargaining equilibrium proposals of all players converge to the same proposal, being the (generalized) fixed point of the function equal to one minus the cumulative distribution function.

For the general case, consider a sequence of continuation probabilities converging to one. The induced sequence of bargaining equilibria converges to some limit, which we call the limit equilibrium. It is not necessarily the case that limit equilibrium proposals of players are all the same. The configuration of limit equilibrium proposals depends on the partition of the set of players into absorbing sets and transient states, based on the proposer selection process. Starting from any transient state, the proposer selection process eventually enters one of the absorbing sets. The probability to enter a given absorbing set starting from a given transient state is called the absorption probability. The proposer selection process determines a unique stationary distribution on each absorbing set. This stationary distribution determines a generalized fixed point in a similar way as illustrated for the case of time-invariant recognition probabilities.

The limit equilibrium proposals can therefore be described as follows:

(A) Players in the same absorbing set make the same proposal.

(B) The proposal of a player in an absorbing set is the related generalized fixed point.

(C) The proposal of a player in a transient state is the weighted average of the absorbing set proposals, with weights given by the absorption probabilities.

It follows from conditions (A) and (C) above that if there is only one absorbing set, then all players make the same limit equilibrium proposal. In particular, if the transition matrix of the proposer selection process is irreducible, the only absorbing set is the entire set of players. Also, if the recognition probabilities are time-invariant, the only absorbing set is the set of players with strictly positive recognition probabilities. Condition (B) provides a simple procedure to calculate limit equilibrium proposals. 
The median voter theorem (Black, 1958) identifies the median of the players' ideal points as the alternative chosen. The median voter outcome has been given non-cooperative foundations in Banks and Duggan [1] and Cho and Duggan [9], who present a bargaining model similar to ours, but with unanimous approval replaced by approval by the majority of players. Cho and Duggan [9] show under very weak conditions that the median voter outcome corresponds to the limit subgame perfect equilibrium of such a bargaining process. Surprisingly, the median voter outcome does not depend on the specifics of the proposer selection process, under the proviso that each player's recognition probability has a strictly positive lower bound, a sharp contrast to our limit equilibrium proposals. When the cumulative distribution function related to the players' ideal points coincides with the cumulative distribution function induced by the proposer selection process, for instance when all players are recognized according to a uniform time-invariant probability distribution, we can show that our limit equilibrium proposals are always closer to the midpoint of the two most extreme ideal points than the median voter outcome.

The Nash bargaining solution is the solution that maximizes the sum over the players of the logarithms of their gains with respect to the disagreement payoff. Nash [25] shows that the axioms of scale invariance, symmetry, efficiency, and independence of irrelevant alternatives uniquely determine this solution for the case of two players. The Nash bargaining solution has also been given non-cooperative underpinnings. Nash [26] is the starting point of what is known today as the Nash program, and provides a non-cooperative demand game whose equilibrium corresponds to the Nash bargaining solution for the case of two players. Also for the two-player case, Binmore, Rubinstein and Wolinsky demonstrate that the limit equilibrium corresponding to the alternating offers bargaining procedure of Rubinstein [28] corresponds to the Nash bargaining solution.

Extensions of such a result to the multiple-player case are also available. Krishna and Serrano [20] and Chae and Yang [7] provide alternative extensions of the alternating offers procedure to the multiple-player case and show that in the limit the Nash bargaining solution results. These authors relax the requirement of unanimous agreement and study so-called exit games. For the bargaining procedure as used in this paper, Britz, Herings, and Predtetchinski [5] show that under weak conditions the limit equilibrium corresponds to the weighted Nash bargaining solution, with weights given by the stationary distribution corresponding to the proposer selection process. This result extends the results by Miyakawa [24] and Laruelle and Valenciano [21], who also obtain convergence to the Nash bargaining solution for more specific proposer selection processes and stronger assumptions on the space of alternatives.

A natural conjecture is therefore that our limit equilibrium can alternatively be described as an appropriately defined weighted Nash bargaining solution. Notice, however, that all the papers that give non-cooperative support to the Nash bargaining solution make the assumption of strict comprehensiveness of the set of feasible payoff vectors. Such an assumption does not hold in the case of one-dimensional bargaining. We will argue that our limit equilibrium does NOT correspond to an appropriately defined Nash bargaining solution.

Imai and Salonen [15] introduce the concept of the representative Nash bargaining solution for the situation of two-sided bargaining. In a two-sided bargaining problem the alternatives are represented by points in the interval and the players are divided into two groups with diametrically opposite preferences. The authors provide an axiomatization of the representative Nash bargaining solution and the non-cooperative characterization of it as a limit of stationary equilibria in a game of bargaining when the probability of the breakdown of negotiations vanishes. Unlike Imai and Salonen [15], we allow for players to have intermediate preferences. 
The paper has been organized as follows. Section 2 introduces the model and the notion of bargaining equilibrium. In Section 3 we show that each bargaining equilibrium is a subgame perfect equilibrium in stationary strategies, and conversely that each subgame perfect equilibrium in stationary strategies is essentially a bargaining equilibrium. In Section 4 we characterize bargaining equilibria by means of a specific system of equations and prove that for each value of the continuation probability below one there is a unique bargaining equilibrium. Section 5 analyzes bargaining equilibria in a number of special cases, including the case of time-invariant recognition probabilities and a deterministically rotating scheme of proposers. Section 6 presents two results on stochastic matrices that are needed to show the main result of the paper, which is the full characterization of the limit equilibrium and its relation to the generalized fixed point of the function generated by the stationary distribution of the proposer selection process as presented in Section 7. Section 8 studies the relationship between limit equilibrium proposals, the median voter outcome, and the Nash bargaining solution. Section 9 concludes the paper.

\section{Bargaining equilibrium}

We study an environment where the set of available alternatives or social states is represented by the unit interval. A finite set of players $N$ has to choose one alternative from the set $Z=$ $[0,1]$. The implementation of an alternative $z \in[0,1]$ leads to payoffs $u_{i}(z)=1-\left|z-p_{i}\right|$ for player $i$. Thus $p_{i}$ is the ideal point of player $i$, and player $i$ 's payoff decreases linearly with the distance between the ideal point and the alternative. We assume that there are players for which 0 , respectively 1 , are the ideal points. We use the notation $i_{0}$ for a player with $p_{i_{0}}=0$ and $i_{1}$ for a player with $p_{i_{1}}=1$. Our results can be generalized to the case where the set of possible alternatives is a smaller set that does not contain some of the ideal points. In this case one should replace the proposals we derive by projections of these proposals on the smaller set.

Given a continuation probability $\delta \in[0,1)$, we define the bargaining game $\Gamma=\Gamma(\delta)$ as follows. The game $\Gamma$ is a dynamic game of almost perfect information. The game starts in period $t=0$. In each period $t$ nature selects a player to make a proposal and the selected player proposes one of the alternatives from $Z$. Then all players respond to the proposal. Each player can either reject or accept it. The players respond to a proposal sequentially, the order of the responses being history-independent. For simplicity, we assume that all players respond to the proposal, including the proposer himself. If the responders unanimously agree to a proposal, the game ends and the proposal is implemented. As soon as the first rejection occurs, time period $t+1$ begins with probability $\delta$ and negotiations break-down with probability $1-\delta$ and the disagreement outcome results. $^{2}$

If an alternative $z$ is implemented, the payoff to player $i$ is $u_{i}(z)$. The payoff of the disagreement outcome and of perpetual disagreement is zero for every player. Players have von Neumann-Morgenstern utility functions.

The selection of proposers is determined by a Markov process on $N$ as in Kalandrakis [16]. The transition probability from state $k$ to state $i$ is $\pi(i \mid k)$. When player $k$ makes a proposal in period $t$, and negotiations do not break down, then with probability $\pi(i \mid k)$ player $i$ will be selected to make a proposal in period $t+1$. Transition probabilities are collected in the matrix $\Pi$, with $\pi(i \mid k)$ in row $k$ and column $i$. In period $t=0$ the process is initialized by an arbitrary probability distribution on the set $N$. Our analysis carries over to exogenously given Markov

\footnotetext{
2 One can reinterpret $\delta$ as the common discount factor.
} 
processes that are defined on a larger state space. All one has to do is to redefine the set of players as the states of the Markov process and associate the utility function of the proposing player to the state.

Two important special cases of the proposer selection process are time-invariant recognition probabilities and deterministic recognition rules. Recognition probabilities are time-invariant if there is a probability distribution $\mu$ on $N$ such that $\pi(i \mid k)=\mu(i)$ for all $(i, k) \in N \times N$. Recognition is deterministic if there is a function $r: N \rightarrow N$ such that $\pi(i \mid k)$ equals one if $i=r(k)$ and zero otherwise. The function $r$ is called a recognition rule. When $N$ consists of players $i_{0}$ and $i_{1}$ only and recognition is deterministic with the rule $r\left(i_{0}\right)=i_{1}$ and $r\left(i_{1}\right)=i_{0}$, we obtain the famous model of alternating offers bargaining of Rubinstein [28] as a special case.

We will analyze subgame perfect equilibria in stationary strategies. In a stationary strategy a player chooses the same action at information sets with an identical continuation game. A stationary strategy of player $i$ consists of a proposal $x_{i} \in[0,1]$ and a collection $A_{i}$ of acceptance sets $A_{i \mid k} \subset[0,1]$ for $k \in N$. The set $A_{i \mid k}$ is the set of proposals of player $k$ that are accepted by player $i$. The stationary strategy $\left(x_{i}, A_{i}\right)$ therefore determines a unique behavioral strategy $\sigma_{i}$. A stationary strategy profile is a pair $(x, A)$, where $x=\left(x_{i}\right)_{i \in N}$ and $A=\left(A_{i}\right)_{i \in N}$.

A tuple of collections of acceptance sets $A$ induces the social acceptance set $X_{k}=\bigcap_{i \in N} A_{i \mid k}$ for $k \in N$, i.e. the set of proposals by player $k$ that are unanimously accepted. We write $X=$ $\left(X_{k}\right)_{k \in N}$.

Consider the case where all players make proposals that belong to the respective social acceptance set. Then there is no delay before a proposal is accepted. Such a strategy profile $(x, A)$ is called a no-delay strategy profile. A no-delay strategy profile $(x, A)$ induces a matrix of continuation payoffs $Y$ with element $y_{i \mid k}$ in row $k$ and column $i$ defined by

$$
y_{i \mid k}=\sum_{j \in N} \pi(j \mid k) u_{i}\left(x_{j}\right) .
$$

The continuation payoff $y_{i \mid k}$ is the expected payoff to player $i$ conditional on the proposal made by player $k$ being rejected and the next period being reached. Player $i$ should reject a proposal $x_{k}$ by player $k$ if $u_{i}\left(x_{k}\right)<\delta y_{i \mid k}$. These considerations motivate the definition of a bargaining equilibrium below.

Definition 2.1. A stationary strategy profile $(x, A)$ is a bargaining equilibrium of $\Gamma$ if

$$
\begin{aligned}
& A_{i \mid k}=\left\{z \in Z \mid u_{i}(z) \geqslant \delta y_{i \mid k}\right\} \quad \text { for each }(i, k) \in N \times N \text {, } \\
& y_{i \mid k}=\sum_{j \in N} \pi(j \mid k) u_{i}\left(x_{j}\right) \quad \text { for each }(i, k) \in N \times N \text {, } \\
& X_{k}=\bigcap_{i \in N} A_{i \mid k} \quad \text { and } \quad X_{k} \neq \emptyset \quad \text { for each } k \in N \text {, } \\
& x_{k}=\arg \max _{z \in X_{k}} u_{k}(z) \quad \text { for each } k \in N .
\end{aligned}
$$

In a bargaining equilibrium no delay ever occurs as all equilibrium proposals $x_{k}$ lie in the respective social acceptance sets $X_{k}$. The number $y_{i \mid k}$ is the equilibrium continuation payoff to player $i$ after a proposal of player $k$ has been rejected. Player $i$ accepts a proposal $x_{k}$ of player $k$ if and only if the payoff of $x_{k}$ is at least as high as the continuation probability times the continuation payoff. 


\section{Subgame perfect equilibria in stationary strategies}

In this section we show that a bargaining equilibrium is a subgame perfect equilibrium in stationary strategies. Moreover, we show the converse result that if $(x, A)$ is a subgame perfect equilibrium in stationary strategies, then there is a bargaining equilibrium where all equilibrium proposals, all social acceptance sets, and all continuation payoffs coincide with those induced by $(x, A)$. In particular, any subgame perfect equilibrium in stationary strategies has the immediate agreement property. As a consequence of the results in this section, we may restrict our analysis of $\Gamma$ to a study of its bargaining equilibria.

The results of this section do not depend on the specific functional form of the utility functions assumed in Section 2. All results of this section hold if (a) the set of alternatives is $Z=[0,1]$, (b) the utility functions $u_{i}: Z \rightarrow \mathbb{R}$ are continuous, concave, single-peaked and non-negative on $Z$, and (c) there is a player $i_{0}$ whose ideal point equals 0 and a player $i_{1}$ whose ideal point is 1 .

Theorem 3.1. A bargaining equilibrium $\sigma=(x, A)$ of $\Gamma$ is a subgame perfect equilibrium in stationary strategies.

The proof of Theorem 3.1 is straightforward. It is sufficient to show that no player has a profitable one-shot deviation from the strategy $\sigma$. It then follows from the one-shot deviation property that there are no profitable deviations from the strategy $\sigma$.

Let $\sigma=(x, A)$ be a stationary strategy profile in the game $\Gamma$. Let $N_{a}=\left\{k \in N \mid x_{k} \in X_{k}\right\}$ denote the set of players whose proposal is accepted and $N_{r}=\left\{k \in N \mid x_{k} \notin X_{k}\right\}$ denote the set of players whose proposal is rejected. We now compute the matrix $Y$ of continuation payoffs associated with $\sigma$. The matrix $Y$ contains $y_{i \mid k}$ in row $k$ and column $i$. If nature chooses a proposer $k$ from the set $N_{a}$, the payoff to player $i$ is $u_{i}\left(x_{k}\right)$, while if the proposer is chosen from $N_{r}$, then the payoff to player $i$ is $\delta y_{i \mid k}$. We have thus a system of equations

$$
y_{i \mid k}=\sum_{j \in N_{a}} \pi(j \mid k) u_{i}\left(x_{j}\right)+\delta \sum_{j \in N_{r}} \pi(j \mid k) y_{i \mid j} \quad \text { for all }(i, k) \in N \times N .
$$

Given a subset $S$ of $N$, let $1_{S}$ be the vector in $\mathbb{R}^{N}$ with coordinates $i \in S$ equal to 1 and coordinates $i \in N \backslash S$ equal to 0 . Let $\Omega(S)$ be a square matrix with entries indexed by elements of the set $N \times N$. The only non-zero entries of $\Omega(S)$ are diagonal entries corresponding to the elements of the set $S$. These entries are equal to one. The matrix $\Omega(N)$ is equal to the identity matrix and is also denoted by $I$. Let $u(x)$ denote the matrix with element $u_{i}\left(x_{k}\right)$ in row $k$ and column $i$. Then the above system can be written in vector-matrix notation as

$$
Y=\Pi \Omega\left(N_{a}\right) u(x)+\delta \Pi \Omega\left(N_{r}\right) Y .
$$

We can now solve for the continuation payoffs:

$$
Y=\Lambda u(x), \quad \text { where } \Lambda=\left[I-\delta \Pi \Omega\left(N_{r}\right)\right]^{-1} \Pi \Omega\left(N_{a}\right) .
$$

The matrix $\left[I-\delta \Pi \Omega\left(N_{r}\right)\right]$ is invertible and has a non-negative inverse, because the spectral radius of $\delta \Pi \Omega\left(N_{r}\right)$ is at most $\delta<1$. The matrix $\Lambda$ is therefore non-negative. Columns of $\Lambda$ corresponding to players in $N_{r}$ are equal to zero. The matrix $\Lambda$ equals the matrix $\Pi$ if $N_{r}$ is empty and has all entries equal to zero if $N_{a}$ is empty.

Furthermore, the sum of the entries of the matrix $\Lambda$ in any given row is at most 1 , that is $\Lambda 1_{N} \leqslant 1_{N}$. To prove this inequality, consider the following chain of equations: 


$$
\begin{aligned}
{\left[I-\delta \Pi \Omega\left(N_{r}\right)\right][I-\Lambda] 1_{N} } & =\left[I-\delta \Pi \Omega\left(N_{r}\right)-\Pi \Omega\left(N_{a}\right)\right] 1_{N} \\
& =\left[I-\delta \Pi \Omega\left(N_{r}\right)-\delta \Pi \Omega\left(N_{a}\right)-(1-\delta) \Pi \Omega\left(N_{a}\right)\right] 1_{N} \\
& =\left[I-\delta \Pi-(1-\delta) \Pi \Omega\left(N_{a}\right)\right] 1_{N} \\
& =1_{N}-\delta 1_{N}-(1-\delta) \Pi 1_{N_{a}} \\
& =(1-\delta)\left(1_{N}-\Pi 1_{N_{a}}\right) \\
& =(1-\delta)\left(\Pi 1_{N}-\Pi 1_{N_{a}}\right) \\
& =(1-\delta) \Pi 1_{N_{r}} .
\end{aligned}
$$

The equation in the third line follows from the fact that $\Omega\left(N_{a}\right)+\Omega\left(N_{r}\right)=I$. The equation in the fourth line uses that $\Pi 1_{N}=1_{N}$ and $\Omega(S) 1_{N}=1_{S}$. The equation in the sixth line again uses the fact that $\Pi 1_{N}=1_{N}$. The equation in the last line uses the equation $1_{N_{a}}+1_{N_{r}}=1_{N}$. Premultiplying the obtained equality by the matrix $\left[I-\delta \Pi \Omega\left(N_{r}\right)\right]^{-1}$ yields

$$
1_{N}-\Lambda 1_{N}=(1-\delta)\left[I-\delta \Pi \Omega\left(N_{r}\right)\right]^{-1} \Pi 1_{N_{r}},
$$

which is non-negative because $\left[I-\delta \Pi \Omega\left(N_{r}\right)\right]^{-1}$ is non-negative. This proves that $\Lambda 1_{N} \leqslant 1_{N}$.

With $\lambda(j \mid k)$ denoting the entry in row $k$ and column $j$ of the matrix $\Lambda$, we can write the continuation payoff as

$$
y_{i \mid k}=\sum_{j \in N_{a}} \lambda(j \mid k) u_{i}\left(x_{j}\right)
$$

Theorem 3.2. Suppose $\delta \in[0,1)$. Let $\sigma=(x, A)$ be a stationary subgame perfect equilibrium of the game $\Gamma$ with continuation payoffs $Y$ and social acceptance sets $X$. Then there exists a bargaining equilibrium $(x, B)$ with continuation payoffs $Y$ and social acceptance sets $X$.

Proof. For a subset $S$ of $Z$ we write Int $S$ to denote the interior of $S$ in the Euclidean topology of the real line. Define the following sets:

$$
\begin{aligned}
& B_{i \mid k}=\left\{z \in Z \mid u_{i}(z) \geqslant \delta y_{i \mid k}\right\} \quad \text { and } \quad B_{k}=\bigcap_{i \in N} B_{i \mid k}, \\
& C_{i \mid k}=\left\{z \in Z \mid u_{i}(z)>\delta y_{i \mid k}\right\} \quad \text { and } \quad C_{k}=\bigcap_{i \in N} C_{i \mid k} .
\end{aligned}
$$

Step 1. We prove that Int $B_{k} \subset C_{k} \subset X_{k} \subset B_{k}$ for each $k$.

To prove the first inclusion, observe that Int $B_{i \mid k} \subset C_{i \mid k}$ because the function $u_{i}$ is concave and single-peaked. It follows that Int $B_{k} \subset \bigcap_{i \in N} \operatorname{Int} B_{i \mid k} \subset \bigcap_{i \in N} C_{i \mid k}=C_{k}$.

To prove the second inclusion let $z$ be an element of $C_{k}$. Suppose $z$ is not an element of $X_{k}$. Let $i$ be the last player in the response order such that $z$ is not an element of the individual acceptance set $A_{i \mid k}$.

Consider any node $h$ of the game $\Gamma$ where player $i$ has to react to the proposal $z$ of player $k$. If all players follow the profile of strategies $\sigma$, then $z$ is rejected with player $i$ being the last player in the response sequence to reject $z$. Thus under strategy $\sigma$ the payoff at node $h$ to player $i$ is $\delta y_{i \mid k}$.

Consider a strategy $\bar{\sigma}_{i}$ of player $i$ that coincides with $\sigma_{i}$ on all nodes of the game $\Gamma$ except node $h$ where it assigns that player $i$ accept the proposal $z$. Because all players following $i$ in the response sequence accept $z$, playing $\bar{\sigma}_{i}$ against $\sigma$ in the subgame that starts at node $h$ leads to a 
payoff $u_{i}(z)$ for player $i$. Since $z \in C_{i \mid k}$ we know that $u_{i}(z)>\delta y_{i \mid k}$. Thus $\bar{\sigma}_{i}$ is a profitable oneshot deviation from $\sigma$ at node $h$ for player $i$. This contradicts the hypothesis that $\sigma$ is a subgame perfect equilibrium.

To prove the third inclusion let $z$ be an element of $X_{k}$ and suppose $z$ is not an element of $B_{k}$. Let $i$ be any player such that $z$ is not an element of the set $B_{i \mid k}$. Then $u_{i}(z)<\delta y_{i \mid k}$.

Consider any node $h$ of the game $\Gamma$ where player $i$ has to react to the proposal $z$ of player $k$. If all players follow the profile of strategies $\sigma$, then $z$ is accepted, resulting in payoff $u_{i}(z)$ for player $i$. Consider a strategy $\bar{\sigma}_{i}$ for player $i$ that coincides with $\sigma_{i}$ on all nodes of the game $\Gamma$ except node $h$ where it assigns that player $i$ reject the proposal $z$. Playing $\bar{\sigma}_{i}$ against $\sigma$ results in a payoff of $\delta y_{i \mid k}$ for player $i$. Thus $\bar{\sigma}_{i}$ is a profitable one-shot deviation from $\sigma$ at node $h$. This contradiction proves the second inclusion.

Step 2. We prove that the set $C_{k}$ is non-empty for each $k$.

Fix some $k \in N$. Let $N^{0}=\left\{i \in N \mid y_{i \mid k}=0\right\}$ and $N^{+}=\left\{i \in N \mid y_{i \mid k}>0\right\}$. Since all utility functions $u_{i}$ are assumed to be concave, single-peaked and non-negative on $[0,1]$, they are positive on $(0,1)$. We have therefore the inclusion

$$
(0,1) \subset \bigcap_{i \in N^{0}} C_{i \mid k}
$$

Let $\lambda(N \mid k)$ denote the sum of all entries in row $k$ of $\Lambda$. Suppose first that $\lambda(N \mid k)=0$. Then row $k$ of $\Lambda$ is zero, and therefore $y_{i \mid k}=0$ for all $i \in N$. Thus $N^{0}=N$ and we have the inclusion $(0,1) \subset C_{k}$. In this case the claim has been proven.

Suppose now that $\lambda(N \mid k)>0$. Define the element $z$ of $Z$ by

$$
z=\frac{\sum_{j \in N_{a}} \lambda(j \mid k) x_{j}}{\lambda(N \mid k)}
$$

For each $i \in N^{+}$we then have the following inequalities:

$$
u_{i}(z) \geqslant \frac{\sum_{j \in N_{a}} \lambda(j \mid k) u_{i}\left(x_{j}\right)}{\lambda(N \mid k)}=\frac{y_{i \mid k}}{\lambda(N \mid k)} \geqslant y_{i \mid k}>\delta y_{i \mid k},
$$

where the first inequality follows from the concavity of $u_{i}$, the second inequality from the fact that $\lambda(N \mid k) \leqslant 1$, and the third inequality from the fact that $\delta<1$ and $y_{i \mid k}>0$. We have thus established the inclusion

$$
z \in \bigcap_{i \in N^{+}} C_{i \mid k}
$$

Each $C_{i \mid k}$ is an open subset of $Z$. Since the set $N^{+}$is finite, $\bigcap_{i \in N^{+}} C_{i \mid k}$ is also an open subset of $Z$. As we have just shown, it is a non-empty set. Being a non-empty open subset of $Z$, it has to have a non-empty intersection with the interior of $Z$. The result follows.

Step 3. We prove that $N_{a}=N$.

Suppose not. Take some $k$ in $N_{r}$. We show that player $k$ has a profitable one-shot deviation from $\sigma$ at any node $h$ where he has to make a proposal.

Under strategy $\sigma$, player $k$ makes a proposal $x_{k}$ that is rejected, resulting in a payoff of $\delta y_{k \mid k}$ for player $k$. Take an arbitrary point $z$ in $C_{k}$ and consider a strategy that agrees with $\sigma_{k}$ on all nodes of the game $\Gamma$ except node $h$, where it assigns that player $k$ make a proposal $z$. Since $C_{k} \subset X_{k}$, the alternative $z$ is unanimously accepted, resulting in the payoff $u_{k}(z)$. Since $z \in C_{k \mid k}$, we have the inequality $u_{k}(z)>\delta y_{k \mid k}$. Proposing $z$ at node $h$ is therefore a profitable one-shot deviation from $\sigma$. 
Step 4. We show that $x_{k}=\arg \max _{z \in X_{k}} u_{k}(z)$ for all $k \in N$.

From Step 3 we know that $x_{k} \in X_{k}$ for all $k$. If $x_{k}$ does not maximize the function $u_{k}$ on the set $X_{k}$, there exists an alternative $z \in X_{k}$ such that $u_{k}(z)>u_{k}\left(x_{k}\right)$. But then player $k$ has a profitable one-shot deviation from $\sigma$ at any node $h$ where he has to make a proposal, namely to propose the alternative $z$. Since $z \in X_{k}$, the alternative $z$ is unanimously accepted, resulting in the payoff $u_{k}(z)$. This contradicts the fact that $\sigma$ is a subgame perfect equilibrium.

Step 5. We show that $X_{k}=B_{k}$ for all $k \in N$.

We know from Step 1 that Int $B_{k} \subset X_{k} \subset B_{k}$ and from Step 2 that Int $B_{k} \neq \emptyset$. Since $B_{k}$ is a convex set, the set $X_{k}$ is also convex and its closure equals $B_{k}$. It is therefore sufficient to show that both $a=\inf X_{k}$ and $b=\sup X_{k}$ are contained in $X_{k}$.

From Step 4 we know that $x_{i_{0}}$ maximizes the utility function $u_{i_{0}}$ of player $i_{0}$ on $X_{k}$, and $x_{i_{1}}$ maximizes the utility function $u_{i_{1}}$ of player $i_{1}$ on $X_{k}$. But $u_{i_{0}}$ is a decreasing function and $u_{i_{1}}$ is an increasing function. Thus, we must have that $x_{i_{0}}=a$ and $x_{i_{1}}=b$. This proves the claim.

Step 6. We prove that $(x, B)$ is a bargaining equilibrium with continuation payoffs $Y$ and social acceptance sets $X$.

Eqs. (1) and (3) hold by definition of $B_{i \mid k}$ and $B_{k}=X_{k}$. We know from Step 3 that $N_{a}=N$. Therefore, the matrix $\Lambda$ equals $\Pi$ and $Y=\Pi u(x)$, which is Eq. (2). Step 4 yields Eq. (4).

\section{A characterization of bargaining equilibria}

In this section we show that each game $\Gamma$ has a unique bargaining equilibrium. Together with Theorems 3.1 and 3.2 of Section 3, this implies that all subgame perfect equilibria in stationary strategies have the same equilibrium proposals, equilibrium utilities, and social acceptance sets.

Herings and Predtetchinski [13] analyze a one-dimensional bargaining model where players can be clustered in two coalitions, one group having $z=0$ as the most preferred point, the other $z=1$. In that special case, there is no need to rely on stationarity of strategies to obtain a similar uniqueness result as in this section, and an analysis of subgame perfect equilibria suffices to obtain the desired result. Within the more general setting of this paper, it is possible to construct examples with multiple subgame perfect equilibria, as is also common in the literature on the extension of the Rubinstein model to the $n$-player case, see Herrero [14] and Haller [12].

Consider a bargaining equilibrium $(x, A)$ with continuation payoffs $Y$ and social acceptance sets $X$. Since the utility functions $u_{i}$ are concave, all individual acceptance sets are closed intervals. We shall use the notation $\left[a_{i \mid k}^{-}, a_{i \mid k}^{+}\right]$to denote the individual acceptance set $A_{i \mid k}$ of player $i$ for the proposals of player $k$. The social acceptance set $X_{k}$ for the proposals of player $k$ is also a closed interval, denoted by $\left[x_{k}^{-}, x_{k}^{+}\right]$.

We now present the two main theorems of this section. Theorem 4.1 is a characterization of bargaining equilibria by means of a system of equations in terms of the equilibrium proposals and the social acceptance sets. Theorem 4.2 states that the bargaining equilibrium is unique.

The system (5)-(6) below is referred to as the characteristic system of equations:

$$
\begin{aligned}
& x^{-}=\delta \Pi x, \quad x^{+}=(1-\delta) 1_{N}+\delta \Pi x, \\
& x_{k}= \begin{cases}x_{k}^{-}, & \text {if } p_{k} \leqslant x_{k}^{-}, \\
p_{k}, & \text { if } x_{k}^{-} \leqslant p_{k} \leqslant x_{k}^{+}, \quad \text { for all } k \in N . \\
x_{k}^{+}, & \text {if } x_{k}^{+} \leqslant p_{k},\end{cases}
\end{aligned}
$$

Theorem 4.1. Let $(x, A)$ be a bargaining equilibrium with social acceptance sets $X_{k}=\left[x_{k}^{-}, x_{k}^{+}\right]$. Then the triple $\left(x, x^{-}, x^{+}\right)$is a solution to the characteristic system of equations. Conversely, 
suppose the triple $\left(x, x^{-}, x^{+}\right)$is a solution to the characteristic system of equations. Then there exists a bargaining equilibrium with equilibrium proposal profile $x$ and social acceptance sets $\left[x_{k}^{-}, x_{k}^{+}\right]$.

Theorem 4.2. There is a unique bargaining equilibrium.

Cardona and Ponsatí [6] show the uniqueness of bargaining equilibrium in the onedimensional model of bargaining assuming that the utility functions are concave, single-peaked and symmetric around the peak, that is for utility functions of the form $u_{i}(z)=f_{i}\left(\left|z-p_{i}\right|\right)$, however they restrict their attention to the case where the proposer is chosen according to some deterministically rotating scheme. While the proof Theorem 4.2 relies on the specific functional form of the utility functions assumed in this paper, its contribution is to cover the case where the identity of the proposer follows a general Markov process. It might be possible to show the uniqueness of bargaining equilibrium for the class of concave, single-peaked and symmetric around the peak utility functions as in Cardona and Ponsatí [6] and the general Markov recognition process. It is clear, however, that the uniqueness result cannot be extended the class of concave single-peaked preferences, an example of a game having a continuum of equilibria being given in Kalandrakis [18].

The remainder of the section is devoted to the proof of these two results. If $x_{k}$ is an equilibrium proposal of player $k$ in a bargaining equilibrium, then it is the point of $X_{k}$ closest to the ideal point $p_{k}$, whence Eq. (6). To derive Eq. (5) we rely on Propositions 4.1 and 4.2 below. Proposition 4.1 essentially says that the players whose ideal points are 0 and 1 determine all social acceptance sets. Player $i_{1}$ whose ideal point is 1 determines the lower endpoint of each social acceptance set, while player $i_{0}$ with ideal point 0 determines the upper endpoint.

Proposition 4.1. Suppose the tuple (x, A,Y,X) satisfies Eqs. (1), (2), and (3). Let $A_{i \mid k}=$ $\left[a_{i \mid k}^{-}, a_{i \mid k}^{+}\right]$and $X_{k}=\left[x_{k}^{-}, x_{k}^{+}\right]$. Then

(a) $x_{k}^{-}=\max _{i \in N}\left\{a_{i \mid k}^{-}\right\}$and $x_{k}^{+}=\min _{i \in N}\left\{a_{i \mid k}^{+}\right\}$,

(b) if $p_{i} \leqslant p_{j}$, then $a_{i \mid k}^{-} \leqslant a_{j \mid k}^{-}$and $a_{i \mid k}^{+} \leqslant a_{j \mid k}^{+}$,

(c) the equations $x_{k}^{-}=a_{i_{1} \mid k}^{-}$and $x_{k}^{+}=a_{i_{0} \mid k}^{+}$hold, and

(d) $X_{k}=A_{i_{0} \mid k} \cap A_{i_{1} \mid k}$.

Proof. Claim (a) follows directly from Eq. (3).

To prove claim (b), notice that $a_{i \mid k}^{-}=\max \left\{0, z_{i \mid k}^{-}\right\}$and $a_{i \mid k}^{+}=\min \left\{1, z_{i \mid k}^{+}\right\}$, where

$$
z_{i \mid k}^{-}=p_{i}-\left(1-\delta y_{i \mid k}\right) \quad \text { and } \quad z_{i \mid k}^{+}=p_{i}+\left(1-\delta y_{i \mid k}\right) .
$$

As $\max \{0, \cdot\}$ and $\min \{1, \cdot\}$ are non-decreasing functions, it is sufficient to show that $z_{i \mid k}^{-} \leqslant z_{j \mid k}$ and $z_{i \mid k}^{+} \leqslant z_{j \mid k}^{+}$whenever $p_{i} \leqslant p_{j}$.

For all real $z$ and $\dot{z}$ the inequality $|z|-|\dot{z}| \leqslant|z-\dot{z}|$ holds. Consequently, for each $i$ and $j$ in $N$ and each $z$ in $Z$ we have $u_{i}(z)-u_{j}(z)=\left|p_{i}-z\right|-\left|p_{j}-z\right| \leqslant\left|p_{i}-p_{j}\right|$, so $\left|u_{i}(z)-u_{j}(z)\right| \leqslant$ $\left|p_{i}-p_{j}\right|$. It follows that

$$
\left|y_{i \mid k}-y_{j \mid k}\right| \leqslant \sum_{l \in N} \pi(l \mid k)\left|u_{i}\left(x_{l}\right)-u_{j}\left(x_{l}\right)\right| \leqslant\left|p_{i}-p_{j}\right| .
$$

Now suppose that $p_{i} \leqslant p_{j}$. Then 


$$
\begin{aligned}
& z_{i \mid k}^{-}-z_{j \mid k}^{-}=p_{i}-p_{j}+\delta\left(y_{i \mid k}-y_{j \mid k}\right) \leqslant p_{i}-p_{j}+\delta\left|p_{i}-p_{j}\right|=(1-\delta)\left(p_{i}-p_{j}\right) \leqslant 0, \\
& z_{i \mid k}^{+}-z_{j \mid k}^{+}=p_{i}-p_{j}-\delta\left(y_{i \mid k}-y_{j \mid k}\right) \leqslant p_{i}-p_{j}+\delta\left|p_{i}-p_{j}\right|=(1-\delta)\left(p_{i}-p_{j}\right) \leqslant 0 .
\end{aligned}
$$

Claim (c) follows immediately from (a) and (b).

Claim (d) follows from (c) and from the fact that $A_{i_{0} \mid k}=\left[0, a_{i_{0} \mid k}^{+}\right]$and $A_{i_{1} \mid k}=\left[a_{i_{1} \mid k}^{-}, 1\right]$.

Proposition 4.2. Suppose the tuple (x, A, Y, X) satisfies Eq. (1), (2), and (3). Let $X_{k}=\left[x_{k}^{-}, x_{k}^{+}\right]$. Then $x^{-}=\delta \Pi x$ and $x^{+}=(1-\delta) 1_{N}+\delta \Pi x$.

Proof. We already know from Proposition 4.1 that $x_{k}^{-}=a_{i_{1} \mid k}^{-}$and $x_{k}^{+}=a_{i_{0} \mid k}^{+}$. The utility function of player $i_{1}$ is $u_{i_{1}}(z)=z$. Thus $y_{i_{1} \mid k}=(\Pi x)_{k}$. It follows that $a_{i_{1} \mid k}^{-}=\delta(\Pi x)_{k}$. The utility function of player $i_{0}$ is $u_{i_{0}}(z)=1-z$. Therefore, $y_{i_{0} \mid k}=1-(\Pi x)_{k}$. It follows that $a_{i_{0} \mid k}^{+}=1-\delta+\delta(\Pi x)_{k}$. The result follows.

Now the proof of Theorem 4.1 is immediate. Suppose $(x, A)$ is a bargaining equilibrium with continuation payoffs $Y$ and social acceptance sets $X=\left(\left[x_{k}^{-}, x_{k}^{+}\right]\right)_{k \in N}$. Eq. (6) then holds because $x_{k}$ is the point of the social acceptance set $\left[x_{k}^{-}, x_{k}^{+}\right]$closest to the point $p_{k}$. Eq. (5) holds by Proposition 4.2. Conversely, suppose the triple $\left(x, x^{-}, x^{+}\right)$is a solution to the characteristic system of equations. Define $Y, A$, and $X$ by Eqs. (2), (1), and (3), respectively, and let $X_{k}=$ $\left[\dot{x}_{k}^{-}, \dot{x}_{k}^{+}\right]$. We must show that Eq. (4) holds and that $\dot{x}^{-}=x^{-}$and $\dot{x}^{+}=x^{+}$. Now, since the tuple $(x, A, Y, X)$ satisfies Eqs. (1), (2), and (3), Proposition 4.2 implies that $\dot{x}^{-}=\delta \Pi x$ and $\dot{x}^{+}=(1-\delta) 1_{N}+\delta \Pi x$. Eq. (5) now implies that $\dot{x}^{-}=x^{-}$and $\dot{x}^{+}=x^{+}$. Eq. (6) implies Eq. (4). Thus $(x, A)$ is a bargaining equilibrium.

Now we turn to the proof of the uniqueness result. Given $i \in N$ and $z \in Z$, let $h_{i}(z)$ be the point of the interval $[\delta z, 1-\delta+\delta z]$ closest to the point $p_{i}$. Define the function $F: Z^{N} \rightarrow Z^{N}$ by letting $F_{i}(x)=h_{i}\left((\Pi x)_{i}\right)$ for each $x$ in $Z^{N}$. It follows from Theorem 4.1 that $x$ is an equilibrium proposal profile in a bargaining equilibrium if and only if $x$ is a fixed point of the function $F$. Proposition 4.3 below shows that the function $F$ is a contraction with respect to the norm on $Z^{N}$ given by $\|x\|=\max \left|x_{i}\right|$. It then follows that $F$ has a unique fixed point, thus establishing Theorem 4.2.

\section{Proposition 4.3.}

(a) For each $i \in N$, each $z$ and $\dot{z}$ in $Z,\left|h_{i}(z)-h_{i}(\dot{z})\right| \leqslant \delta|z-\dot{z}|$.

(b) For each $x$ in $\mathbb{R}^{N},\|\Pi x\| \leqslant\|x\|$.

(c) For each $x$ and $\dot{x}$ in $Z^{N},\|F(x)-F(\dot{x})\| \leqslant \delta\|x-\dot{x}\|$.

Proof. To prove claim (a) write the function $h_{i}$ as

$$
h_{i}(z)= \begin{cases}1-\delta+\delta z, & \text { if } z \in Z_{1}=\left[0,\left(p_{i}+\delta-1\right) / \delta\right], \\ p_{i}, & \text { if } z \in Z_{2}=\left[\left(p_{i}+\delta-1\right) / \delta, p_{i} / \delta\right], \\ \delta z, & \text { if } z \in Z_{3}=\left[p_{i} / \delta, 1\right] .\end{cases}
$$

The function $h_{i}$ is affine with a slope of $\delta$ on $Z_{1}$ and $Z_{3}$ and it is a constant on $Z_{2}$. The result follows.

To prove claim (b), observe that for each $k \in N$ we have the inequalities

$$
\left|(\Pi x)_{k}\right|=\left|\sum_{i \in N} \pi(i \mid k) x_{i}\right| \leqslant \sum_{i \in N} \pi(i \mid k)\left|x_{i}\right| \leqslant \sum_{i \in N} \pi(i \mid k)\|x\|=\|x\| .
$$


The result follows.

To prove claim (c) we compute

$$
\begin{aligned}
\left|F_{i}(x)-F_{i}(\dot{x})\right| & =\left|h_{i}\left((\Pi x)_{i}\right)-h_{i}\left((\Pi \dot{x})_{i}\right)\right| \\
& \leqslant \delta\left|(\Pi x)_{i}-(\Pi \dot{x})_{i}\right| \\
& =\delta\left|(\Pi(x-\dot{x}))_{i}\right| \\
& \leqslant \delta\|x-\dot{x}\| .
\end{aligned}
$$

The result follows.

\section{Some special cases}

Motivated by Theorem 4.1, we refer to the solution $\left(x, x^{-}, x^{+}\right)$of the system (5)-(6) of characteristic equations as a bargaining equilibrium.

\subsection{Time-invariant recognition probabilities}

When the recognition probabilities are time-invariant, the continuation payoff $y_{i \mid k}$ of player $i$ does not depend on the identity of the proposer, so $y_{i \mid k}$ is independent of $k$. In particular, a rejection of a proposal by player $k$ leads to the same continuation payoff as a rejection of a proposal by player $j$. It follows that the individual acceptance set $A_{i \mid k}$ of player $i$ for the proposals of player $k$ does not depend on $k$. Therefore, also the social acceptance sets $X_{k}$ do not depend on $k$ and are all equal to some interval $[a, b]$.

Let the time-invariant recognition probabilities be given by the probability distribution $\mu$ on $N$. The characteristic system of equations then simplifies as follows:

$$
\begin{aligned}
& a=\delta \mu x, \quad b=1-\delta+\delta \mu x, \\
& x_{k}= \begin{cases}a, & \text { if } p_{k} \leqslant a, \\
p_{k}, & \text { if } a \leqslant p_{k} \leqslant b, \quad \text { for all } k \in N, \\
b, & \text { if } b \leqslant p_{k},\end{cases}
\end{aligned}
$$

where $\mu x=\sum_{i \in N} \mu(i) x_{i}$. The equilibrium proposals are given by Fig. 1 below. Notice that the equilibrium proposal $x_{i}$ is non-decreasing in $p_{i}$. An interesting feature of a bargaining equilibrium in the model with time-invariant recognition probabilities is that it is fully characterized by two numbers, namely $a$ and $b$, the endpoints of the common social acceptance set.

Given a continuation probability $\delta \in[0,1)$, we let $(x(\delta), a(\delta), b(\delta))$ denote the unique bargaining equilibrium in a model with time-invariant recognition probabilities $\mu$. The limit of the bargaining equilibrium as $\delta$ approaches one, if it exists, is called the limit equilibrium. Now we compute the limit equilibrium for the case of time-invariant recognition probabilities. In Section 7 we compute the limit equilibrium for the general case.

Definition 5.1. A point $z \in Z$ is said to be a generalized fixed point of a non-increasing function $f: \mathbb{R} \rightarrow Z$ if $f(z+\varepsilon) \leqslant z \leqslant f(z-\varepsilon)$ for all $\varepsilon>0$.

It is clear that each non-increasing function has at most one generalized fixed point.

Define a cumulative distribution function $F: \mathbb{R} \rightarrow Z$ by the equation

$$
F(z)=\mu\left(\left\{i \in N \mid p_{i} \leqslant z\right\}\right) .
$$




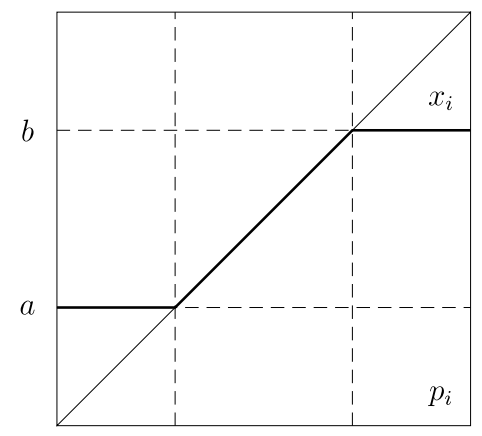

Fig. 1. Equilibrium proposal $x_{i}$ in the case of time-invariant recognition probabilities.

Thus $F(z)$ is the mass of players with ideal point belonging to the interval $[0, z]$. It is clear that $F$ is a non-decreasing function. Theorem 5.1 characterizes limit equilibria as the generalized fixed point of the function $1-F$. The proof of the theorem relies on the following result.

Proposition 5.1. Let $(x, a, b)$ be a bargaining equilibrium in a model with time-invariant recognition probabilities. Then $1-F(b) \leqslant \mu x \leqslant 1-F(a)$.

Proof. Define the elements $z^{-}$and $z^{+}$of $Z^{N}$ as follows:

$$
z_{i}^{-}=\left\{\begin{array}{ll}
a, & \text { if } p_{i} \in[0, b], \\
b, & \text { otherwise }
\end{array} \quad \text { and } \quad z_{i}^{+}= \begin{cases}a, & \text { if } p_{i} \in[0, a] \\
b, & \text { otherwise }\end{cases}\right.
$$

It is obvious that $z^{-} \leqslant x \leqslant z^{+}$. Therefore $\mu z^{-} \leqslant \mu x \leqslant \mu z^{+}$. Now, we use Eq. (7) to compute:

$$
\begin{aligned}
& \mu z^{-}=F(b) a+(1-F(b)) b=(1-\delta)(1-F(b))+\delta \mu x, \\
& \mu z^{+}=F(a) a+(1-F(a)) b=(1-\delta)(1-F(a))+\delta \mu x .
\end{aligned}
$$

This yields the desired inequalities $1-F(b) \leqslant \mu x \leqslant 1-F(a)$.

Theorem 5.1. The limits $\lim _{\delta \uparrow 1} x_{i}(\delta)$ for $i \in N, \lim _{\delta \uparrow 1} a(\delta)$, and $\lim _{\delta \uparrow 1} b(\delta)$ exist. All limits are equal to the unique generalized fixed point of the function $1-F$.

Proof. Let $\delta_{n}$ be a sequence in $[0,1)$ converging to 1 . We must show that the sequences $x_{i}\left(\delta_{n}\right)$ for each $i \in N, a\left(\delta_{n}\right)$ and $b\left(\delta_{n}\right)$ converge to the generalized fixed point of the function $1-F$.

Without loss of generality, assume that the sequences $x_{i}\left(\delta_{n}\right)$ for each $i \in N, a\left(\delta_{n}\right)$, and $b\left(\delta_{n}\right)$ converge. From Eq. (7) of the characteristic system for the model with time-invariant recognition probabilities we know that $b\left(\delta_{n}\right)-a\left(\delta_{n}\right)=1-\delta_{n}$, so the sequences $a\left(\delta_{n}\right)$ and $b\left(\delta_{n}\right)$ converge to the same limit. Denote this common limit by $z$. Since $a\left(\delta_{n}\right) \leqslant x_{i}\left(\delta_{n}\right) \leqslant b\left(\delta_{n}\right)$ the sequence $x_{i}\left(\delta_{n}\right)$ converges to $z$ for each $i \in N$ and so does the sequence $\mu x\left(\delta_{n}\right)$.

To prove that $z$ is generalized fixed point of the function $1-F$, let $\varepsilon>0$. Then for $n$ large enough

$$
z-\varepsilon \leqslant a\left(\delta_{n}\right) \leqslant b\left(\delta_{n}\right) \leqslant z+\varepsilon .
$$

Because $F$ is a non-decreasing function,

$$
F(z-\varepsilon) \leqslant F\left(a\left(\delta_{n}\right)\right) \leqslant F\left(b\left(\delta_{n}\right)\right) \leqslant F(z+\varepsilon) .
$$


Applying Proposition 5.1, we find that

$$
1-F(z+\varepsilon) \leqslant 1-F\left(b\left(\delta_{n}\right)\right) \leqslant \mu x\left(\delta_{n}\right) \leqslant 1-F\left(a\left(\delta_{n}\right)\right) \leqslant 1-F(z-\varepsilon) .
$$

Taking the limit we obtain the desired inequality

$$
1-F(z+\varepsilon) \leqslant z \leqslant 1-F(z-\varepsilon) .
$$

The result follows because $\varepsilon>0$ is arbitrary.

As we see from Theorem 5.1, in the model with time-invariant recognition probabilities, the common social acceptance set collapses to a point as the continuation probability approaches one. As a consequence, the limit equilibrium proposals of all players are the same.

With general Markov recognition probabilities, different proposers face different social acceptance sets. While it is still true that a social acceptance set for the proposal of a given proposer collapses to a point, this point is in general different for different proposers and Theorem 5.1 does not simply carry over to the general case.

\subsection{Symmetric recognition probabilities}

In this subsection we assume that $N \subset Z$ and $p_{i}=i$ for all $i \in N$. The player set $N$ is said to be symmetric (around 1/2) if $1-i \in N$ whenever $i \in N$. Suppose $N$ is symmetric. The recognition probabilities $\Pi$ are said to be symmetric (around 1/2) if $\pi(1-i \mid k)=\pi(i \mid k)$ for all $i$ and $k$ in $N$.

Proposition 5.2. Assume $N \subset Z$ and $p_{i}=i$ for all $i \in N$. Assume the player set $N$ and the recognition probabilities $\Pi$ are symmetric. Let $x_{i}^{-}=\delta / 2$ and $x_{i}^{+}=1-\delta / 2$ for all $i \in N$ and let $x$ be given by $E q$. (8) with $a=\delta / 2$ and $b=1-\delta / 2$. Then $\left(x, x^{-}, x^{+}\right)$is a bargaining equilibrium.

Proof. We verify that the tuple $\left(x, x^{-}, x^{+}\right)$satisfies Eqs. (5)-(6).

It is clear from Fig. 1 that Eq. (6) holds. Since $b=1-a$, the function $x$ is symmetric in the sense that $x_{1-i}=1-x_{i}$ for all $i \in N$. Therefore, for any $k \in N$,

$$
\begin{aligned}
2(\Pi x)_{k} & =\sum_{i \in N} \pi(i \mid k) x_{i}+\sum_{i \in N} \pi(1-i \mid k) x_{1-i} \\
& =\sum_{i \in N} \pi(i \mid k) x_{i}+\sum_{i \in N} \pi(i \mid k) x_{1-i} \\
& =\sum_{i \in N} \pi(i \mid k)=1,
\end{aligned}
$$

where the first equality holds because $N$ is symmetric, the second one holds because $\Pi$ is symmetric, and the third one holds because $x$ is symmetric. We see that $\Pi x$ is identically equal to 1/2. It follows that Eq. (5) holds.

Thus, when $N$ and $\Pi$ are symmetric, the bargaining equilibrium resembles the one in the case of time-invariant recognition probabilities in that all players face the same social acceptance set. As $\delta$ approaches one, the equilibrium proposals of all players converge to $1 / 2$. Notice however, that, unlike the case of time-invariant recognition probabilities, the expected payoffs $y_{i \mid k}$ and the individual acceptance sets $A_{i \mid k}$ depend on $k$. 


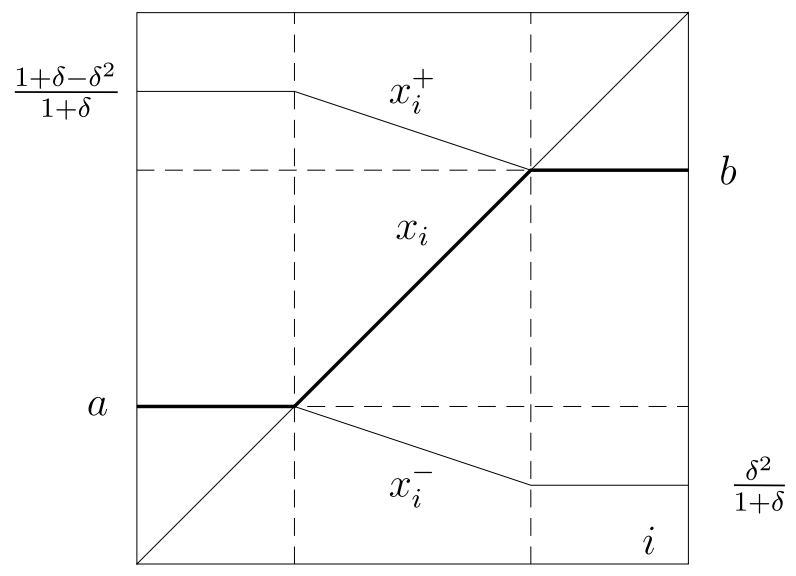

Fig. 2. Illustration of $x, x^{-}$and $x^{+}$as in claim (b) of Proposition 5.3.

\subsection{Deterministic recognition rules}

Let $r: N \rightarrow N$ be a recognition rule. Eq. (5) can then be rewritten as

$$
x_{i}^{-}=\delta x_{r(i)}, \quad \text { and } \quad x_{i}^{+}=1-\delta+\delta x_{r(i)} \quad \text { for all } i \in N .
$$

Claim (a) of Proposition 5.3 below presents the case where a player, once chosen to be the proposer, remains the proposer for the rest of the game. The result is, not surprisingly, that each player proposes his own ideal point. Claim (b) contains the model of Rubinstein [28] as a special case. It deals with the case where any player $i$, once chosen to be the proposer, alternates with player $1-i$ in being the proposer for the rest of the game.

Proposition 5.3. Assume $N \subset Z$ and $p_{i}=i$ for all $i \in N$. Consider a game $\Gamma$ with a deterministic recognition rule $r$ :

(a) Let $r$ be the identity. The bargaining equilibrium $\left(x, x^{-}, x^{+}\right)$is given by $x_{i}^{-}=\delta i, x_{i}^{+}=$ $1-\delta+\delta i$ and $x_{i}=i$ for all $i \in N$.

(b) Suppose the set $N$ is symmetric around $1 / 2$ and $r(i)=1-i$. Let $a=\delta /(1+\delta)$ and $b=$ $1 /(1+\delta)$. Then the bargaining equilibrium $\left(x, x^{-}, x^{+}\right)$is given by

$$
\begin{aligned}
& x_{i}= \begin{cases}a, & \text { if } i \leqslant a, \\
i, & \text { if } a \leqslant i \leqslant b, \\
b, & \text { if } b \leqslant i,\end{cases} \\
& x_{i}^{-}=\left\{\begin{array}{ll}
\delta /(1+\delta), & \text { if } i \leqslant a, \\
\delta-\delta i, & \text { if } a \leqslant i \leqslant b, \\
\delta^{2} /(1+\delta), & \text { if } b \leqslant i,
\end{array} \quad x_{i}^{+}= \begin{cases}\left(1+\delta-\delta^{2}\right) /(1+\delta), & \text { if } i \leqslant a, \\
1-\delta i, & \text { if } a \leqslant i \leqslant b, \\
b, & \text { if } b \leqslant i .\end{cases} \right.
\end{aligned}
$$

Proof. Claim (a) is obvious. Consider claim (b). Fig. 2 illustrates the bargaining equilibrium. It is clear from the figure that $x_{i}$ is the point of $\left[x_{i}^{-}, x_{i}^{+}\right]$closest to $i$, so that Eq. (6) holds. Notice that because $b=1-a, x$ is symmetric in the sense that $x_{1-i}=1-x_{i}$ for all $i \in N$. Using this property, it is straightforward to verify that Eq. (9) holds as well. 


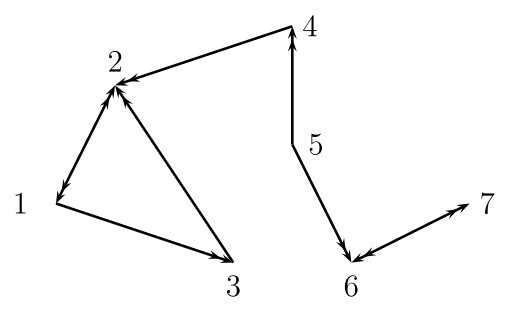

Fig. 3. Transition probabilities. There is a double arrow from $j$ to $i$ if the one-step probability $\pi(i \mid j)$ of transition from $j$ to $i$ is non-zero. The depicted Markov process has two absorbing sets, $\{1,2,3\}$ and $\{6,7\}$. The states 4 and 5 are transient states. Starting from state 5 there is a non-zero probability to enter either absorbing set, while starting from state 4 the process enters the absorbing set $\{1,2,3\}$ with probability 1 .

In part (a) of Proposition 5.3, the equilibrium proposals do not depend on $\delta$.

Fig. 2 illustrates the bargaining equilibrium in part (b) of Proposition 5.3. Observe that both $x_{i}^{-}$and $x_{i}^{+}$are non-increasing functions of $i$. The equilibrium proposal $x_{i}$ is non-decreasing in $i$. The equilibrium proposal of each player converges to $1 / 2$ as $\delta$ approaches one.

\section{Two results on stochastic matrices}

This section provides the mathematical results needed to analyze the asymptotic behavior of bargaining equilibria. Given a set $S \subset N$, let $S^{\mathrm{c}}$ denote the set $N \backslash S$. Let $\pi(S \mid i)=\sum_{j \in S} \pi(j \mid i)$.

\subsection{Absorbing sets and transient states}

The state $j$ is said to lead to state $i$, written as $j \rightarrow i$, if $i=j$ or if there exists a natural number $n$ such that $\pi^{n}(i \mid j)>0$. The states $j$ and $i$ communicate, written as $j \leftrightarrow i$, if $j \rightarrow i$ and $i \rightarrow j$. The relation $\leftrightarrow$ is an equivalence relation. An equivalence class $S$ of the relation $\leftrightarrow$ is called an absorbing set if $\pi(S \mid i)=1$ for all $i \in S$. A state $i$ is said to be transient if it is not an element of any absorbing set. We let $\mathcal{A}$ be the collection of absorbing sets and $D$ be the set of transient states. Fig. 3 illustrates.

Given a subset $S$ of $N$, let $\Pi_{S}$ denote the restriction of $\Pi$ to the states in $S$. Notice that if $S$ is an absorbing set, then $\Pi_{S}$ is a stochastic matrix, i.e. the elements in each row of $\Pi_{S}$ add up to 1 . A stochastic matrix $\Pi$ is said to be irreducible if $N$ is the only absorbing set. If $S$ is an absorbing set, then the stochastic matrix $\Pi_{S}$ is irreducible.

Given an absorbing set $S$, an element $\mu_{S}$ of $\mathbb{R}^{S}$ is said to be a stationary distribution on $S$ if $\mu_{S} \Pi_{S}=\mu_{S}$ and $\sum_{i \in S} \mu_{S}(i)=1$. By convention, $\mu_{S}$ is a row vector. For each absorbing set $S$ there exists a unique stationary distribution on $S$, see Theorem 4.1 in Seneta [29]. It will be convenient to extend $\mu_{S}$ to an element of $\mathbb{R}^{N}$ by letting $\mu_{S}(i)=0$ for all $i \in S^{\mathrm{c}}$.

\subsection{The convergence of the matrix $\Psi(\delta)$}

Given $\delta \in[0,1)$, define the matrix $\Psi=\Psi(\delta)$ as follows:

$$
\Psi=(1-\delta) \sum_{n=0}^{\infty} \delta^{n} \Pi^{n}=(1-\delta)(I-\delta \Pi)^{-1} .
$$

The following is a well-known result. 
Theorem 6.1. Let $\Pi$ be a stochastic irreducible matrix. Let $\mu_{N}$ denote the unique stationary distribution on $N$. As $\delta$ approaches one from below, each row of the matrix $\Psi(\delta)$ converges to $\mu_{N}$.

We shall use the following straightforward corollary of Theorem 6.1.

Corollary 6.1. Let $S$ be an absorbing set and let $\mu_{S}$ be the corresponding stationary distribution on $S$. As $\delta$ approaches one from below, each row of the matrix $\Psi(\delta)$ corresponding to a state in $S$ converges to $\mu_{S}$.

\subsection{The Perron-Frobenius Theorem and absorption probabilities}

We state a version of the Perron-Frobenius Theorem for a stochastic irreducible matrix that will be sufficient for our purposes (see Theorem 1.5 in Seneta [29]).

Theorem 6.2. Let $\Pi$ be a stochastic irreducible matrix. An element $x$ in $\mathbb{R}^{N}$ satisfies the equation $\Pi x=x$ if and only if it is constant on $N$.

Using Theorem 6.2 we obtain a characterization of the eigenspace of a general stochastic matrix associated with eigenvalue one.

Starting from any transient state $i \in D$, the Markov process eventually leaves $D$ and enters one of the absorbing sets where it stays forever. Given an absorbing set $S$ and a transient state $i$, let $\varphi(S \mid i)$ denote the probability for the process to eventually enter the set $S$ given the initial state $i$. Let $\varphi(S \mid D)$ be the column vector of absorption probabilities $\varphi(S \mid i)$ as the index $i$ ranges over all transient states. Let $\pi(S \mid D)$ be the column vector of probabilities $\pi(S \mid i)$ as the index $i$ ranges over all transient states. The result quoted below combines Theorems 4.3 and 4.4 from Seneta [29].

Theorem 6.3. Let $\Pi$ be a stochastic matrix. Then the matrix $I_{D}-\Pi_{D}$ is invertible. Furthermore, $\varphi(S \mid D)=\left[I_{D}-\Pi_{D}\right]^{-1} \pi(S \mid D)$ for each absorbing set $S$.

The following corollary provides a characterization of the eigenspace of a general stochastic matrix $\Pi$ associated with eigenvalue one.

Corollary 6.2. Let $\Pi$ be a stochastic matrix. An element $x$ of $\mathbb{R}^{N}$ satisfies the equation $\Pi x=x$ if and only if (a) the vector $x$ is constant on each absorbing set, and (b) for each state $i \in D$

$$
x_{i}=\sum_{S \in \mathcal{A}} \varphi(S \mid i) x_{S},
$$

where $x_{S}$ is the value of $x$ on an absorbing set $S$.

Proof. Suppose $\Pi x=x$.

Let $S$ be an absorbing set and let $x_{\mid S}$ denote the restriction of $x$ to $S$. It follows that $\Pi_{S} x_{\mid S}=x_{\mid S}$. Since $S$ is an absorbing set, the matrix $\Pi_{S}$ is a stochastic irreducible matrix. By Theorem 6.2, the vector $x_{\mid S}$ is constant on the set $S$.

Let $x_{S}$ denote the value of $x$ on $S$ and $x_{\mid D}$ denote the restriction of $x$ to $D$. Then the equations corresponding to the transient states can be written as 


$$
\sum_{S \in \mathcal{A}} \pi(S \mid D) x_{S}+\Pi_{D} x_{\mid D}=x_{\mid D}
$$

Solving the system, we obtain

$$
x_{\mid D}=\left[I_{D}-\Pi_{D}\right]^{-1} \sum_{S \in \mathcal{A}} \pi(S \mid D) x_{S}=\sum_{S \in \mathcal{A}} \varphi(S \mid D) x_{S} .
$$

The result follows. The converse direction is now easy to prove.

Consider a Markov process as in Fig. 3. If the vector $x$ satisfies the equation $\Pi x=x$, then $x_{1}=x_{2}=x_{3}$ and $x_{6}=x_{7}$, since $\{1,2,3\}$ and $\{6,7\}$ are the absorbing sets of the process. Furthermore, $x_{4}=x_{1}$, because, starting from state 4 , the process enters the absorbing set $\{1,2,3\}$ with probability 1 . Finally, $x_{5}$ is a convex combination of $x_{1}$ and $x_{6}$ with weights equal to the respective absorption probabilities.

\section{The limit equilibrium}

In this section we study the limit of the bargaining equilibrium when the continuation probability tends to one. The limit is shown to exist and is called the limit equilibrium. We characterize the limit equilibrium as a generalized fixed point of a particular function. We study under what conditions all players make the same proposal in the limit equilibrium.

Given $x \in Z^{N}$, let $B(x)=\left\{i \in N \mid x_{i}<p_{i}\right\}$. Notice that $B(x) \supset B(\dot{x})$ whenever $x \leqslant \dot{x}$.

Proposition 7.1. Let $\left(x, x^{-}, x^{+}\right)$be a bargaining equilibrium. Then $\Psi 1_{B^{-}} \leqslant x \leqslant \Psi 1_{B^{+}}$where $B^{-}=B\left(x^{+}\right)$and $B^{+}=B\left(x^{-}\right)$.

Proof. Define the elements $z^{-}$and $z^{+}$of $Z^{N}$ as follows:

$$
z_{k}^{-}=\left\{\begin{array}{ll}
x_{k}^{-}, & \text {if } p_{k} \leqslant x_{k}^{+}, \\
x_{k}^{+}, & \text {otherwise, }
\end{array} \quad \text { and } \quad z_{k}^{+}= \begin{cases}x_{k}^{-}, & \text {if } p_{k} \leqslant x_{k}^{-}, \\
x_{k}^{+}, & \text {otherwise }\end{cases}\right.
$$

Using Eq. (6) of the characteristic system, it is easy to see that $z^{-} \leqslant x \leqslant z^{+}$. Since $\Pi$ preserves the relation $\leqslant$, we also have $\Pi z^{-} \leqslant \Pi x \leqslant \Pi z^{+}$, and, for each natural $n$

$$
\Pi^{n} z^{-} \leqslant \Pi^{n} x \leqslant \Pi^{n} z^{+}
$$

The set of players $k \in N$ such that $x_{k}^{+}<p_{k}$ is by definition $B^{-}=B\left(x^{+}\right)$. For each $k$ in $B^{-}$ we have $z_{k}^{-}=x_{k}^{+}$and for each $k$ in the complement of $B^{-}$we have $z_{k}^{-}=x_{k}^{-}$. By Eq. (5) of the characteristic system $x^{-}=\delta \Pi x$ and $x^{+}=(1-\delta) 1_{N}+\delta \Pi x$. Recall that $1_{B^{-}}$is a vector in $\mathbb{R}^{N}$ with coordinates in $B^{-}$equal to 1 and other coordinates equal to zero. We can therefore rewrite $z^{-}$as

$$
z^{-}=\delta \Pi x+(1-\delta) 1_{B^{-}} \quad \text { and similarly } \quad z^{+}=\delta \Pi x+(1-\delta) 1_{B^{+}} .
$$

Applying $\Pi^{n}$ to each side of the system of equations, we obtain

$$
\Pi^{n} z^{-}=\delta \Pi^{n+1} x+(1-\delta) \Pi^{n} 1_{B^{-}} \quad \text { and } \quad \Pi^{n} z^{+}=\delta \Pi^{n+1} x+(1-\delta) \Pi^{n} 1_{B^{+}} .
$$

Now we have the following chain of inequalities: 


$$
\begin{aligned}
x & \leqslant z^{+}=\delta \Pi x+(1-\delta) 1_{B^{+}} \\
& \leqslant \delta \Pi z^{+}+(1-\delta) 1_{B^{+}}=\delta^{2} \Pi^{2} x+(1-\delta) 1_{B^{+}}+(1-\delta) \delta \Pi 1_{B^{+}} \\
& \leqslant \cdots \\
& \leqslant \delta^{n+1} \Pi^{n+1} x+(1-\delta) \sum_{i=0}^{n} \delta^{n} \Pi^{n} 1_{B^{+}} .
\end{aligned}
$$

As $n$ goes to infinity, the first term of the last expression vanishes, while the second term converges to $\Psi 1_{B^{+}}$. We thus obtain the upper bound $x \leqslant \Psi 1_{B^{+}}$on $x$. To obtain the lower bound, consider the chain of inequalities

$$
\begin{aligned}
x & \geqslant z^{-}=\delta \Pi x+(1-\delta) 1_{B^{-}} \\
& \geqslant \delta \Pi z^{-}+(1-\delta) 1_{B^{-}}=\delta^{2} \Pi^{2} x+(1-\delta) 1_{B^{-}}+(1-\delta) \delta \Pi 1_{B^{-}} \\
& \geqslant \cdots \\
& \geqslant \delta^{n+1} \Pi^{n+1} x+(1-\delta) \sum_{i=0}^{n} \delta^{n} \Pi^{n} 1_{B^{-}} .
\end{aligned}
$$

Taking the limit as $n$ goes to infinity, we obtain the lower bound $x \geqslant \Psi 1_{B^{-}}$.

For each absorbing set $S$ define $F_{S}: \mathbb{R} \rightarrow Z$ by letting $F_{S}(z)=\mu_{S}\left(\left\{i \in S \mid p_{i} \leqslant z\right\}\right)$. As $F_{S}$ is a non-decreasing function, the function $1-F_{S}$ has a unique generalized fixed point, see Definition 5.1.

Definition 7.1. The limit equilibrium $x^{\ell}$ is the element of $Z^{N}$ satisfying the following conditions: (A) The vector $x^{\ell}$ is constant on each absorbing set $S$, (B) the value $x_{S}^{\ell}$ of $x^{\ell}$ on $S$ is the generalized fixed point of the function $1-F_{S}$, and (C) the value of $x^{\ell}$ on each transient state $i$ is given by

$$
x_{i}^{\ell}=\sum_{S \in \mathcal{A}} \varphi(S \mid i) x_{S}^{\ell} .
$$

The conditions (A) and (C) of Definition 7.1 are equivalent to the requirement that $\Pi x^{\ell}=x^{\ell}$, see Corollary 6.2.

Given a continuation probability $\delta \in[0,1)$, we let $\left(x(\delta), x^{-}(\delta), x^{+}(\delta)\right)$ denote the bargaining equilibrium.

Theorem 7.1. The limits $\lim _{\delta \uparrow 1} x(\delta), \lim _{\delta \uparrow 1} x^{-}(\delta)$, and $\lim _{\delta \uparrow 1} x^{+}(\delta)$ exist. All three limits are equal to $x^{\ell}$.

Proof. Let $\delta_{n}$ be a sequence in $[0,1)$ converging to 1 . We must show that the sequences $x\left(\delta_{n}\right)$, $x^{-}\left(\delta_{n}\right)$, and $x^{+}\left(\delta_{n}\right)$ converge to $x^{\ell}$. Without loss of generality assume that the sequence $x\left(\delta_{n}\right)$ converges to an element $x$ of $Z^{N}$.

The sequence $\Pi x\left(\delta_{n}\right)$ converges to $\Pi x$. By Eq. (5) of the characteristic system, $x^{-}\left(\delta_{n}\right)=$ $\delta_{n} \Pi x\left(\delta_{n}\right)$ and $x^{+}\left(\delta_{n}\right)=\left(1-\delta_{n}\right) 1_{N}+\delta_{n} \Pi x\left(\delta_{n}\right)$. It follows that the sequences $x^{-}\left(\delta_{n}\right)$ and $x^{+}\left(\delta_{n}\right)$ converge to $\Pi x$. Since $x^{-}\left(\delta_{n}\right) \leqslant x\left(\delta_{n}\right) \leqslant x^{+}\left(\delta_{n}\right)$, it then follows that $x\left(\delta_{n}\right)$ converges to $\Pi x$. But we know that $x\left(\delta_{n}\right)$ converges to $x$. Thus $x=\Pi x$. 
We conclude that $x$ is an eigenvector of the matrix $\Pi$ associated with eigenvalue one. We now use Corollary 6.2 in Section 6 that provides a complete description of the eigenspace of the matrix $\Pi$ associated with eigenvalue one. Corollary 6.2 implies that $x$ is constant on each absorbing set and that Eq. (10) holds. It remains to be shown that the value $x_{S}$ of $x$ on each absorbing set $S$ is a generalized fixed point of the function $1-F_{S}$.

We already know that the sequences $x^{-}\left(\delta_{n}\right)$ and $x^{+}\left(\delta_{n}\right)$ converge to $x$. Let $\varepsilon>0$ be given. Since $N$ is a finite set, for $n$ large enough the following inequalities hold:

$$
x-1_{N} \varepsilon \leqslant x^{-}\left(\delta_{n}\right) \leqslant x^{+}\left(\delta_{n}\right) \leqslant x+1_{N} \varepsilon .
$$

It follows that for $n$ large enough we have the inclusions

$$
B\left(x+1_{N} \varepsilon\right) \subset B\left(x^{+}\left(\delta_{n}\right)\right) \subset B\left(x^{-}\left(\delta_{n}\right)\right) \subset B\left(x-1_{N} \varepsilon\right) .
$$

These inclusions imply the inequalities

$$
1_{B\left(x+1_{N} \varepsilon\right)} \leqslant 1_{B\left(x^{+}\left(\delta_{n}\right)\right)} \leqslant 1_{B\left(x^{-}\left(\delta_{n}\right)\right)} \leqslant 1_{B\left(x-1_{N} \varepsilon\right)} .
$$

We then also have the inequalities

$$
\Psi\left(\delta_{n}\right) 1_{B\left(x+1_{N} \varepsilon\right)} \leqslant \Psi\left(\delta_{n}\right) 1_{B\left(x^{+}\left(\delta_{n}\right)\right)} \leqslant x\left(\delta_{n}\right) \leqslant \Psi\left(\delta_{n}\right) 1_{B\left(x^{-}\left(\delta_{n}\right)\right)} \leqslant \Psi\left(\delta_{n}\right) 1_{B\left(x-1_{N} \varepsilon\right)},
$$

where the middle inequalities follow from Proposition 7.1.

Now let $S$ be an absorbing set and let $x_{S}$ be the value of $x$ on $S$. Let $k \in S$ and consider the inequality

$$
\left[\Psi\left(\delta_{n}\right) 1_{B\left(x+1_{N} \varepsilon\right)}\right]_{k} \leqslant x_{k}\left(\delta_{n}\right) \leqslant\left[\Psi\left(\delta_{n}\right) 1_{B\left(x-1_{N} \varepsilon\right)}\right]_{k} .
$$

By Corollary 6.1 in Section 6, row $k$ of the matrix $\Psi\left(\delta_{n}\right)$ converges to $\mu_{S}$ as $n$ goes to infinity. We thus obtain the inequalities

$$
\mu_{S} 1_{B\left(x+1_{N} \varepsilon\right)} \leqslant x_{S} \leqslant \mu_{S} 1_{B\left(x-1_{N} \varepsilon\right)} .
$$

Now, for any set $B \subset N$ we have $\mu_{S} 1_{B}=\mu_{S}(B \cap S)$. Furthermore, $B\left(x+1_{N} \varepsilon\right) \cap S=\{i \in S \mid$ $\left.x_{S}+\varepsilon<p_{i}\right\}$ and $B\left(x-1_{N} \varepsilon\right) \cap S=\left\{i \in S \mid x_{S}-\varepsilon<p_{i}\right\}$. Thus, the preceding inequality can be rewritten as

$$
\mu_{S}\left(\left\{i \in S \mid x_{S}+\varepsilon<p_{i}\right\}\right) \leqslant x_{S} \leqslant \mu_{S}\left(\left\{i \in S \mid x_{S}-\varepsilon<p_{i}\right\}\right) .
$$

The left-hand side equals $1-F_{S}\left(x_{S}+\varepsilon\right)$ and the right-hand side equals $1-F_{S}\left(x_{S}-\varepsilon\right)$. Thus we can once again rewrite the preceding inequality as

$$
1-F_{S}\left(x_{S}+\varepsilon\right) \leqslant x_{S} \leqslant 1-F_{S}\left(x_{S}-\varepsilon\right) .
$$

Since $\varepsilon>0$ is arbitrary, it follows that $x_{S}$ is the generalized fixed point of the function $1-F_{S}$, as desired.

Now we identify some special cases where $x^{\ell}$ is constant on the entire player set $N$. In these cases every player makes the same proposal in the limit equilibrium.

Proposition 7.2. If the proposer selection process has a unique absorbing set, then $x^{\ell}$ is constant on $N$. 
Proof. The result follows directly from the definition of limit equilibrium. The vector $x^{\ell}$ is constant on the absorbing set $S$. Furthermore, starting from each state $i$ outside $S$ the process is absorbed into $S$ with probability 1 , that is $\varphi(S \mid i)=1$.

Proposition 7.3. If the matrix $\Pi$ is irreducible, then $x^{\ell}$ is constant on $N$.

Proof. If the matrix $\Pi$ is irreducible, then $N$ is the only absorbing set.

Proposition 7.4. Suppose the proposer selection process has time-invariant recognition probabilities $\mu$. Then $x^{\ell}$ is constant on $N$ and its value is the generalized fixed point of the function $1-F$, where $F(z)=\mu\left(\left\{i \in N \mid p_{i} \leqslant z\right\}\right)$.

Proof. If $\mu$ are the time-invariant recognition probabilities, then $S=\{i \in N \mid \mu(i)>0\}$ is the only absorbing set, and the stationary distribution corresponding to $S$ is $\mu$. The result now follows from Proposition 7.2 and the definition of $x^{\ell}$.

\section{Comparison with alternative concepts}

This section compares the limit equilibrium outcome with two solutions figuring prominently in the literature, the median voter outcome and the Nash bargaining solution. We assume throughout this section that the proposer selection process has a unique absorbing set $S$ with cumulative distribution function $F_{S}$ induced by the stationary distribution $\mu_{S}$. According to Proposition 7.2, the alternative $z^{\ell}$ satisfying, for every $\varepsilon>0, z^{\ell}+F_{S}\left(z^{\ell}-\varepsilon\right) \leqslant 1 \leqslant z^{\ell}+F_{S}\left(z^{\ell}+\varepsilon\right)$ is proposed by every player in the limit equilibrium.

\subsection{The median voter outcome}

There is an extensive literature related to the problem of choosing an alternative from the unit interval by a population of players with single-peaked preferences. The median voter theorem (Black, 1958) identifies the median of the players' ideal points as the selected alternative.

Let $G$ be the cumulative distribution function of the ideal points of the players, $G(z)=\mid\{i \in$ $\left.N \mid p_{i} \leqslant z\right\}|/| N \mid$. The median voter result states that the group of players selects an alternative $z^{\mathrm{m}}$ that is one of the generalized zero points of the function $G-1 / 2$, i.e. for every $\varepsilon>0$, $G\left(z^{\mathrm{m}}-\varepsilon\right) \leqslant 1 / 2 \leqslant G\left(z^{\mathrm{m}}+\varepsilon\right)$. The set of such points is denoted $Z^{\mathrm{m}}$.

Indeed, when a political candidate proposes some $z^{\mathrm{m}} \in Z^{\mathrm{m}}$, then there is no alternative $z$ that is strictly closer than $z^{\mathrm{m}}$ to the ideal point of more than half of the players. When the number of players is odd, $Z^{\mathrm{m}}$ consists of a single point. When the number of players is even, $Z^{\mathrm{m}}$ is the (possibly degenerate) interval with end points given by the two ideal points in the middle. Notice that unlike $z^{\ell}, z^{\mathrm{m}}$ may not be unique.

Since the functions $F_{S}$ and $G$ are unrelated, there is no general connection between $z^{\ell}$ and $z^{\mathrm{m}}$. Notice that where $z^{\mathrm{m}}$ does not depend on $F_{S}, z^{\ell}$ does not depend on $G$. Suppose, however, that the functions $F_{S}$ and $G$ coincide, which would for instance be the case when recognition probabilities are time-invariant and uniform. We then have the following result.

Theorem 8.1. Assume the proposer selection process has a unique absorbing set $S$ and $F_{S}=G$. There are three mutually exclusive cases to consider: 
If $G(1 / 2)<1 / 2$, then for all $z^{\mathrm{m}} \in Z^{\mathrm{m}}$ it holds that

$$
\frac{1}{2}<z^{\ell} \leqslant z^{\mathrm{m}}
$$

If $\lim _{\varepsilon \downarrow 0} G(1 / 2-\varepsilon) \leqslant 1 / 2$ and $G(1 / 2) \geqslant 1 / 2$, then

$$
\frac{1}{2}=z^{\ell} \in Z^{\mathrm{m}} \text {. }
$$

If $\lim _{\mathcal{\varepsilon} \downarrow 0} G(1 / 2-\varepsilon)>1 / 2$, then for all $z^{\mathrm{m}} \in Z^{\mathrm{m}}$ it holds that

$$
z^{\mathrm{m}} \leqslant z^{\ell}<\frac{1}{2}
$$

Proof. A point $z^{\mathrm{m}} \in Z^{\mathrm{m}}$ satisfies, for every $\varepsilon>0, G\left(z^{\mathrm{m}}-\varepsilon\right) \leqslant 1 / 2 \leqslant G\left(z^{\mathrm{m}}+\varepsilon\right)$. The point $z^{\ell}$ satisfies, for every $\varepsilon>0, z^{\ell}+G\left(z^{\ell}-\varepsilon\right) \leqslant 1 \leqslant z^{\ell}+G\left(z^{\ell}+\varepsilon\right)$.

Assume $G(1 / 2)<1 / 2$. Since $G$ is continuous from the right, it holds that

$$
\lim _{\varepsilon \downarrow 0} G\left(\frac{1}{2}+\varepsilon\right)=G\left(\frac{1}{2}\right)<\frac{1}{2},
$$

so $G(1 / 2+\varepsilon)<1 / 2$ for $\varepsilon$ sufficiently small and $z^{\mathrm{m}}>1 / 2$ for every $z^{\mathrm{m}} \in Z^{\mathrm{m}}$. Now it holds that, for every $z^{\mathrm{m}} \in Z^{\mathrm{m}}$,

$$
\lim _{\varepsilon \downarrow 0}\left(z^{\mathrm{m}}+G\left(z^{\mathrm{m}}+\varepsilon\right)\right)>\frac{1}{2}+\lim _{\varepsilon \downarrow 0} G\left(z^{\mathrm{m}}+\varepsilon\right) \geqslant 1,
$$

so $z^{\ell} \leqslant z^{\mathrm{m}}$. Finally,

$$
\lim _{\varepsilon \downarrow 0}\left(\frac{1}{2}+G\left(\frac{1}{2}+\varepsilon\right)\right)=\frac{1}{2}+G\left(\frac{1}{2}\right)<1,
$$

so $1 / 2<z^{\ell}$.

Assume $\lim _{\varepsilon \downarrow 0} G(1 / 2-\varepsilon) \leqslant 1 / 2$ and $G(1 / 2) \geqslant 1 / 2$. We have that for every $\bar{\varepsilon}>0$,

$$
G\left(\frac{1}{2}-\bar{\varepsilon}\right) \leqslant \lim _{\varepsilon \downarrow 0} G\left(\frac{1}{2}-\varepsilon\right) \leqslant \frac{1}{2} \leqslant G\left(\frac{1}{2}\right) \leqslant G\left(\frac{1}{2}+\bar{\varepsilon}\right),
$$

so $1 / 2 \in Z^{\mathrm{m}}$. Inequalities (11) imply that for every $\bar{\varepsilon}>0$

$$
\frac{1}{2}+G\left(\frac{1}{2}-\varepsilon\right) \leqslant 1 \leqslant \frac{1}{2}+G\left(\frac{1}{2}+\varepsilon\right)
$$

so $z^{\ell}=1 / 2$.

Assume $\lim _{\varepsilon \downarrow 0} G(1 / 2-\varepsilon)>1 / 2$. It is immediate that, for every $z^{\mathrm{m}} \in Z^{\mathrm{m}}, z^{\mathrm{m}}<1 / 2$. Also, for $z^{\mathrm{m}} \in Z^{\mathrm{m}}$,

$$
\lim _{\varepsilon \downarrow 0}\left(z^{\mathrm{m}}+G\left(z^{\mathrm{m}}-\varepsilon\right)\right)<\frac{1}{2}+\lim _{\varepsilon \downarrow 0} G\left(z^{\mathrm{m}}-\varepsilon\right) \leqslant 1,
$$

so it holds that $z^{\ell} \geqslant z^{\mathrm{m}}$. Moreover,

$$
\lim _{\varepsilon \downarrow 0}\left(\frac{1}{2}+G\left(\frac{1}{2}-\varepsilon\right)\right)=\frac{1}{2}+\lim _{\varepsilon \downarrow 0} G\left(\frac{1}{2}-\varepsilon\right)>1,
$$

so $z^{\ell}<1 / 2$. 
The alternative $z^{\ell}$ is equal to $1 / 2$ if and only if it belongs to $Z^{\mathrm{m}}$, i.e. $1 / 2$ is a median voter outcome. If $G(1 / 2)<1 / 2$, then it follows that $z^{\mathrm{m}} \geqslant z^{\ell}>1 / 2$, and if $\lim _{\varepsilon \downarrow 0} G(1 / 2-\varepsilon)>1 / 2$, then $z^{\mathrm{m}} \leqslant z^{\ell}<1 / 2$. Median voter outcomes are more extreme than the alternative selected in the limit equilibrium. The underlying intuition is that the bargaining model requires unanimous agreement and therefore also the agreement of both the players $i_{0}$ and $i_{1}$. This causes a tendency to select alternatives in the middle. For the median voter model, the votes of half of the players are sufficient, and the opinion of the other players can be safely ignored.

Most striking is the case where the ideal point of each player is either 0 or 1 and $G(0)=$ $(1+\varepsilon) / 2$ for some $\varepsilon>0$. Then $Z^{\mathrm{m}}=\{0\}$ and $z^{\ell}=(1-\varepsilon) / 2$. When $\varepsilon$ converges to zero, the limit equilibrium outcome converges to $1 / 2$, whereas the median voter outcome is 0 .

\subsection{The Nash bargaining solution}

The Nash bargaining solution is the solution that maximizes the sum over the players of the logarithms of their gains with respect to the disagreement payoff. Binmore, Rubinstein and Wolinsky demonstrate for the two-player case that the limit equilibrium corresponding to the alternating offers bargaining procedure of Rubinstein [28] corresponds to the Nash bargaining solution. For the bargaining procedure as used in this paper, Britz, Herings, and Predtetchinski [5] show that under weak conditions the limit equilibrium corresponds to the weighted Nash bargaining solution, with weights given by the stationary distribution $\mu_{S}$. A natural conjecture is therefore that our limit equilibrium can alternatively be described as an appropriately defined weighted Nash bargaining solution. We will argue next that when $n \geqslant 3$ our limit equilibrium does NOT correspond to an appropriately defined Nash bargaining solution. The reason for this perhaps counterintuitive result is the following. All the papers that give non-cooperative support to the Nash bargaining solution make the assumption of strict comprehensiveness of the set of feasible payoffs. Such an assumption does not hold in the case of one-dimensional bargaining.

The Nash bargaining solution with weights $\mu_{S}$ is defined as the solution $z^{\mathrm{n}}$ to the program

$$
\max _{z \in(0,1)} \sum_{i \in N} \mu_{i} \ln \left(1-\left|z-p_{i}\right|\right),
$$

where we assume for the sake of simplicity that $\mu_{S}\left(i_{0}\right)>0$ for some player $i_{0}$ with ideal point 0 , and $\mu_{S}\left(i_{1}\right)>0$ for some player $i_{1}$ with ideal point 1 to guarantee interior solutions.

For a given alternative $z$ we define $N^{-}(z)=\left\{i \in N \mid p_{i}<z\right\}$ and $N^{+}(z)=\left\{i \in N \mid p^{i}>z\right\}$. Using the strict concavity of the objective functions, it follows by taking the first derivative at points of differentiability that $z^{\mathrm{n}}$ is given by the unique generalized zero point of the strictly increasing function

$$
H(z)=\sum_{i \in N^{-}(z)} \mu_{i} \frac{1}{1-\left|z-p_{i}\right|}+\sum_{i \in N^{+}(z)} \mu_{i} \frac{-1}{1-\left|z-p_{i}\right|} .
$$

That is for every $\varepsilon>0$ we have that $H\left(z^{\mathrm{n}}-\varepsilon\right)<0<H\left(z^{\mathrm{n}}+\varepsilon\right)$. In the typical case where $z^{\mathrm{n}}$ does not coincide with any of the players' ideal points, it is the exact zero of the function $H$. Thus

$$
\sum_{i \in N^{-}\left(z^{\mathrm{n}}\right)} \frac{\mu_{i}}{1-\left|z^{\mathrm{n}}-p_{i}\right|}=\sum_{i \in N^{+}\left(z^{\mathrm{n}}\right)} \frac{\mu_{i}}{1-\left|z^{\mathrm{n}}-p_{i}\right|} .
$$

In the two-player case the condition that $z^{\mathrm{n}}$ does not coincide with any of the players' ideal points is trivially satisfied. For that case we find that 
Table 1

The Nash bargaining solution in an example.

\begin{tabular}{|c|c|c|c|c|c|c|c|c|c|c|}
\hline$m$ & 1 & 2 & 3 & 4 & 5 & 6 & 7 & 8 & 9 & 10 \\
\hline$\overline{z_{m}^{\mathrm{n}}}$ & 0.500 & 0.500 & 0.446 & 0.412 & 0.389 & 0.372 & 0.359 & 0.349 & 0.340 & 0.333 \\
\hline
\end{tabular}

$$
\frac{\mu_{i_{0}}}{1-z^{\mathrm{n}}}=\frac{\mu_{i_{1}}}{z^{\mathrm{n}}}
$$

so $z^{\mathrm{n}}=\mu_{i_{1}}$. In this case the limit equilibrium does coincide with the Nash bargaining solution, since

$$
z^{\mathrm{n}}+F\left(z^{\mathrm{n}}\right)=\mu_{i_{1}}+\mu_{i_{0}}=1
$$

thereby generalizing the findings of Binmore, Rubinstein, and Wolinsky [3]. We have shown the following result.

Theorem 8.2. If $N$ consists of two players and the proposer selection process has absorbing set $N$, then $z^{\ell}=z^{\mathrm{n}}$.

In the $n$-player case there is no general relationship between the Nash bargaining solution and the other two solution concepts. The characterization of the Nash bargaining solution in (12) cannot be rewritten in a form that depends only on either $F_{S}$ or $G$. In general, it depends on both since the characterization in (12) depends on $G$ via the ideal points $p_{i}$ and it depends on $F_{S}$ via the weights $\mu_{S}$.

As an example, consider the case where $m$ players have an ideal point equal to $0, m$ players have an ideal point at $1 / 2$, and 1 player's ideal point is at 1 . Assume the proposer selection process is such that $F_{S}=G$. It is immediate from Theorem 8.1 that $z^{\ell}=z^{\mathrm{m}}=1 / 2$, independent from $m$. The Nash bargaining solution does depend on $m$ and is denoted by $z_{m}^{\mathrm{n}}$.

It can be verified that for $m=1$ it holds that $z_{m}^{\mathrm{n}}$ is equal to $1 / 2$. For $m \geqslant 2$, the Nash bargaining solution $z_{m}^{\mathrm{n}}$ is characterized by

$$
\frac{m}{1-z_{m}^{\mathrm{n}}}=\frac{m}{\frac{1}{2}+z_{m}^{\mathrm{n}}}+\frac{1}{z_{m}^{\mathrm{n}}}
$$

The unique solution to this equation is given by

$$
z_{m}^{\mathrm{n}}=\frac{\frac{1}{2}(m+1)+\sqrt{\frac{1}{4}(m+1)^{2}}+2(2 m+1)}{2(2 m+1)} .
$$

As an example, it follows that $z_{10}^{\mathrm{n}}=1 / 3$. Table 1 gives an overview of $z_{m}^{\mathrm{n}}$ for $1 \leqslant m \leqslant 10$. It can be proven that $\lim _{m \rightarrow \infty} z_{m}^{\mathrm{n}}=1 / 4$.

If we modify the example to have one player with ideal point equal to $0, m$ players with ideal point equal to $1 / 2$, and $m$ players with ideal point equal to 1 , we obtain $z^{\ell}=z^{\mathrm{m}}=1 / 2 \leqslant z_{m}^{\mathrm{n}}$, with strict inequality if and only if $m \geqslant 3$.

\section{Conclusion}

We have formulated the important problem of how to select an alternative in a onedimensional set of alternatives as a non-cooperative bargaining problem. We consider the case 
where a proposal is only accepted if all players approve of it. Subgame perfect equilibrium in stationary strategies is unique up to unimportant details of individual acceptance sets and is called bargaining equilibrium. We provide a simple characterization of the bargaining equilibrium. In our model, proposers are selected according to a general Markov process. This captures two cases that feature prominently in the literature: the one with time-invariant recognition probabilities and the one with a deterministic proposer selection rule.

We study the limit equilibrium, the limit of a sequence of bargaining equilibria as the continuation probability tends to one. We give an explicit description of the limit equilibrium as the generalized fixed point of a function that is intimately related to the time-invariant distribution of the proposer selection process. All players make the same proposal in the limit equilibrium under a wide variety of specifications of the proposer selection process.

When the proposer selection process has a unique absorbing set $S$ with associated stationary distribution $\mu_{S}$ and cumulative distribution function $F_{S}$, and when we ignore the fact that $F_{S}$ is not continuous, the limit equilibrium outcome is given by $z^{\ell}+F_{S}\left(z^{\ell}\right)=1$. Two other solution concepts that figure prominently in the literature are the median voter outcome $z^{\mathrm{m}}$ and the Nash bargaining solution $z^{\mathrm{n}}$. With $G$ the cumulative distribution function associated to the players' ideal points, again ignoring discontinuities, we have that $G\left(z^{\mathrm{m}}\right)=1 / 2$, whereas the Nash bargaining solution satisfies

$$
\sum_{i \in N^{-}\left(z^{\mathrm{n}}\right)} \frac{\mu_{i}}{1-\left|z^{\mathrm{n}}-p_{i}\right|}=\sum_{i \in N^{+}\left(z^{\mathrm{n}}\right)} \frac{\mu_{i}}{1-\left|z^{\mathrm{n}}-p_{i}\right|} .
$$

We show that the limit equilibrium outcome is closer to the midpoint of the two most extreme ideal points than the median voter outcome when $F_{S}$ and $G$ coincide. Both outcomes are in general not related to the Nash bargaining solution. The only exception is the case with two players, when it holds that $z^{\ell}=z^{\mathrm{n}}$.

\section{References}

[1] J.S. Banks, J. Duggan, A bargaining model of collective choice, Amer. Polit. Sci. Rev. 94 (2000) $73-88$.

[2] D.P. Baron, J.A. Ferejohn, Bargaining in legislatures, Amer. Polit. Sci. Rev. 83 (1989) 1181-1206.

[3] K. Binmore, A. Rubinstein, A. Wolinsky, The Nash bargaining solution in economic modelling, RAND J. Econ. 17 (1986) 176-188.

[4] D. Black, The Theory of Committees and Elections, Cambridge Univ. Press, London, 1958.

[5] V. Britz, P.J.J. Herings, A. Predtetchinski, Non-cooperative support for the Nash bargaining solution, METEOR Research Memorandum 08/18, Maastricht University, 2008.

[6] D. Cardona, C. Ponsatí, Bargaining one-dimensional social choices, J. Econ. Theory 137 (2007) 627-651.

[7] S. Chae, J.-A. Yang, An N-person pure bargaining game, J. Econ. Theory 62 (1994) 86-102.

[8] S.-J. Cho, J. Duggan, Uniqueness of stationary equilibria in a one-dimensional model of bargaining, J. Econ. Theory 113 (2003) 118-130.

[9] S.-J. Cho, J. Duggan, Bargaining foundations of the median voter theorem, J. Econ. Theory 144 (2009) $851-868$.

[10] H. Eraslan, Uniqueness of stationary equilibrium payoffs in the Baron-Ferejohn model, J. Econ. Theory 103 (2002) 11-30.

[11] H. Eraslan, A. Merlo, Majority rule in a stochastic model of bargaining, J. Econ. Theory 103 (2002) 31-48.

[12] H. Haller, Non-cooperative bargaining of $N \geqslant 3$ players, Econ. Letters 22 (1986) 11-13.

[13] P.J.J. Herings, A. Predtetchinski, Sequential share bargaining, METEOR Research Memorandum 07/05, Maastricht University, 2007.

[14] M. Herrero, A strategic bargaining approach to market institutions, PhD Thesis, London School of Economics, London, 1985.

[15] H. Imai, H. Salonen, The representative Nash solution for two-sided bargaining problems, Math. Soc. Sci. 39 (2000) $349-365$. 
[16] T. Kalandrakis, Equilibria in sequential bargaining games as solutions to systems of equations, Econ. Letters 84 (2004) 407-411.

[17] T. Kalandrakis, Proposal rights and political power, Amer. J. Polit. Sci. 50 (2006) 441-448.

[18] T. Kalandrakis, Regularity of pure strategy equilibrium points in a class of bargaining games, Econ. Theory 28 (2006) 309-329.

[19] B. Knight, Estimating the value of proposal power, Amer. Econ. Rev. 95 (2005) 1639-1652.

[20] V. Krishna, R. Serrano, Multilateral bargaining, Rev. Econ. Stud. 63 (1996) 61-80.

[21] A. Laruelle, F. Valenciano, Noncooperative foundations of bargaining power in committees and the Shapley-Shubik index, Games Econ. Behav. 63 (2008) 341-353.

[22] A. Merlo, C. Wilson, A stochastic model of sequential bargaining with complete information, Econometrica 63 (1995) 371-399.

[23] A. Merlo, C. Wilson, Efficient delays in a stochastic model of bargaining, Econ. Theory 11 (1998) 39-55.

[24] T. Miyakawa, Noncooperative foundation of $n$-person asymmetric Nash bargaining solution, Working Paper No. 2006-2, Osaka University of Economics, 2006.

[25] J. Nash, The bargaining problem, Econometrica 18 (1950) 155-162.

[26] J. Nash, Two-person cooperative games, Econometrica 21 (1953) 128-140.

[27] Th. Romer, H. Rosenthal, Political resource allocation, controlled agendas, and the status quo, Public Choice 33 (1978) 27-44.

[28] A. Rubinstein, Perfect equilibrium in a bargaining model, Econometrica 50 (1982) 97-109.

[29] E. Seneta, Non-negative Matrices and Markov Chains, Series in Statistics, Springer-Verlag, Berlin, 2006. 